\begin{tabular}{|c|l|}
\hline Title & Code division multiple access multiuser demodulator by using quantum fluctuations \\
\hline Author(s) & Otsubo, Y osuke; Inoue, Jun-ichi; Nagata, Kenji; Okada, Masato \\
\hline Citation & $\begin{array}{l}\text { Physical Review E, 90(1), 012126 } \\
\text { https://doi.org/L0.1103/PhysRevE.90.012126 }\end{array}$ \\
\hline Issue Date & 201407-24 \\
\hline Doc URL & http://hdl.handle.net/2115/57442 \\
\hline Rights & ○2014 A merican Physical Society \\
\hline Type & article \\
\hline File Information & PhysRevE.90.012126.pdf \\
\hline
\end{tabular}

Instructions for use 


\title{
Code-division multiple-access multiuser demodulator by using quantum fluctuations
}

\author{
Yosuke Otsubo \\ Graduate School of Frontier Sciences, The University of Tokyo, Kashiwa, Chiba 277-5861, Japan \\ Jun-ichi Inoue \\ Graduate School of Information Science and Technology, Hokkaido University, Sapporo 060-0814, Japan \\ Kenji Nagata \\ Graduate School of Frontier Sciences, The University of Tokyo, Kashiwa, Chiba 277-5861, Japan \\ Masato Okada* \\ Graduate School of Frontier Sciences, The University of Tokyo, Kashiwa, Chiba 277-5861, Japan \\ and Brain Science Institute, RIKEN, Wako, Saitama 351-0198, Japan
}

(Received 8 January 2014; published 24 July 2014)

\begin{abstract}
We examine the average-case performance of a code-division multiple-access (CDMA) multiuser demodulator in which quantum fluctuations are utilized to demodulate the original message within the context of Bayesian inference. The quantum fluctuations are built into the system as a transverse field in the infinite-range Ising spin glass model. We evaluate the performance measurements by using statistical mechanics. We confirm that the CDMA multiuser modulator using quantum fluctuations achieve roughly the same performance as the conventional CDMA multiuser modulator through thermal fluctuations on average. We also find that the relationship between the quality of the original information retrieval and the amplitude of the transverse field is somehow a "universal feature" in typical probabilistic information processing, viz., in image restoration, error-correcting codes, and CDMA multiuser demodulation.
\end{abstract}

DOI: 10.1103/PhysRevE.90.012126

\section{INTRODUCTION}

Quantum fluctuations by means of the transverse field have been intensely investigated within the context of combinatorial optimization problems, which induce tunneling instead of thermal jumps between states [1-3]. The algorithm is called quantum annealing (QA) or quantum adiabatic algorithm. QA has been applied to various optimization problems by solving the Schrödinger equation or carrying out quantum Monte Carlo simulations on classical computers. However, what we call a quantum annealer with current superconducting devices has been launched by $D$-wave systems based in British Columbia [4-6]. Taking into account these scientific and technological advances, quantum fluctuations induced by transverse fields could have the potential to provide us with several effective tools for solving combinatorial optimization problems. It is also interesting for us to consider the possible application of the quantum fluctuations to probabilistic information processing with developments in the research field of algorithms by making use of quantum fluctuations. Restoration (decoding) algorithms incorporating transverse fields have recently been investigated in image restoration and Sourlas code, which have both been described with infinite-range spin glass models. The average-case performance of these systems has been analyzed with statistical mechanics in the thermodynamic limit $[7,8]$.

Infinite-range spin glass models have received a lot of attention in recent years in terms of information processing, due to adoption of the framework of Bayesian statistics. For example, the Sherrington-Kirkpatrick (SK) model is closely

\footnotetext{
*okada@k.u-tokyo.ac.jp
}

PACS number(s): 02.50.-r, 05.20.-y, 05.50.+q, 03.67.Ac

linked to error-correcting codes or associative memories in neural networks. The average-case performance of decoding or retrieving has been analytically evaluated using the so-called replica method [9-11]. The so-called hyperparameter in these model systems that corresponds to a noise power in the posterior can be regarded as "temperature" in the Gibbs-Boltzman distribution within the context of statistical physics [12].

A typical example is the code-division multiple-access (CDMA) system that has been recognized as a telecommunication technology that simultaneously enables communication among a huge number of users. It has also been extensively analyzed with the replica method [13,14]. The basic idea behind the CDMA is to modulate the (original) digital signals of multiusers. The digital signals are modulated by assigning a distinct spreading code for each user. Then, the modulated signals are transmitted through noisy channels. The maximizer of the posterior marginals (MPM) estimate is utilized within the context of Bayesian inference to simultaneously demodulate the original bit sequences of multiusers for a given set of outputs from the noisy channels. It has been well known that the MPM estimate enables us to construct an optimal demodulator in the sense that the estimate minimizes the bit-error rate on average. Optimal performance is actually achieved by controlling the temperature so that it has the same value as the noise power in the channel. The relationship between the optimal temperature and the corresponding noise amplitude is referred to as the Nishimori line [12]. For this reason, the MPM estimate is often called finite temperature demodulation.

Quantum-mechanical fluctuations are regarded in the literature on physics, as a counterpart of thermal fluctuations. With this remarkable correspondence in mind, it is naturally expected that the MPM estimate can be extended by means 
of quantum fluctuations. Namely, the amplitude of quantum fluctuations might be controlled to satisfy a similar relationship to the Nishimori line to achieve the best possible demodulation. In fact, quantum MPM (QMPM) has been proposed thus far and performance has been investigated within the context of image restoration and Sourlas code [7,8]. Obviously, one can consider the "mixture" of these two distinct fluctuations. Then, the problem that needs to be clarified is to explore the best possible mixture to minimize the bit-error rate for decoding on average. More naively, we should answer a question of the following type, viz., "Which fluctuations give us a better average-case performance for typical problems in probabilistic information processing?" Thus far, we have confirmed that the decoding performance of the MPM estimate incorporating the transverse field can roughly achieve the same performance as that of the optimal thermal MPM estimate, at least for image restoration and Sourlas code.

We focus on CDMA multiuser demodulation under thermal and quantum fluctuations as an example to analyze the averagecase performance in this research and examine the equivalence between thermal and quantum fluctuations in the literature on the optimal MPM estimate. We then compare performance with that of image restoration and error-correcting codes to clarify the central issue, viz., "universal feature" of the equivalence between thermal and quantum fluctuations in Bayesian MPM estimation. We should mention that the demodulating process in the CDMA system is quite similar to so-called compressed sensing (CS) [15-17]. CS and related techniques have been developed to solve various types of modern problems in engineering such as functional magnetic resonance imaging (f-MRI) and image processing.

This paper is organized as follows. The next section introduces the CDMA system and quantum-mechanical extension. We then explain how we derive equations of states and the average-case performance measurements by using the replica method in Sec. III. Section IV presents our results. The last section is a summary and contains concluding remarks.

\section{FORMULATION}

Let us consider a demodulation problem for a wireless communication by $N$ users communicating in fully synchronous channels. Then, the received signal at the base station is given by

$$
y^{k}=\frac{1}{\sqrt{N}} \sum_{i=1}^{N} \eta_{i}^{k} \xi_{i}+\epsilon^{k},
$$

where $\xi_{i} \in\{-1,1\},(i=1, \ldots, N)$ is the original information and $\eta_{i}^{k} \in\{-1,1\},(k=1, \ldots, K, i=1, \ldots, N)$ is referred to as the spreading code sequences for user $i$. The channel noise $\epsilon^{k}$ is inevitably contained in the received signal information (see Fig. 1). By using the following notations:

$$
\begin{gathered}
\boldsymbol{y}=\left(y^{1}, \ldots, y^{K}\right)^{T}, \quad \xi=\left(\xi_{1}, \ldots, \xi_{N}\right)^{T}, \quad \boldsymbol{\epsilon}=\left(\epsilon^{1}, \ldots, \epsilon^{K}\right)^{T}, \\
\mathbf{H}=\left(\begin{array}{cccc}
\eta_{1}^{1} & \eta_{2}^{1} & \cdots & \eta_{N}^{1} \\
\eta_{1}^{2} & \eta_{2}^{2} & \ldots & \eta_{N}^{2} \\
\cdots & \ldots & \ldots & \cdots \\
\eta_{1}^{K} & \eta_{2}^{K} & \ldots & \eta_{N}^{K}
\end{array}\right),
\end{gathered}
$$

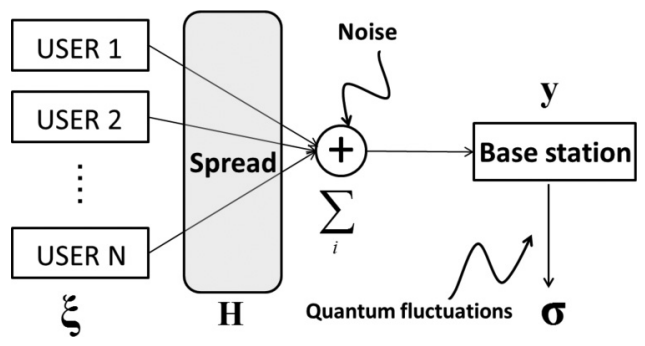

FIG. 1. CDMA multiuser demodulation with quantum fluctuations.

the received signal (1) can be rewritten as

$$
\boldsymbol{y}=\frac{1}{\sqrt{N}} \mathbf{H} \boldsymbol{\xi}+\boldsymbol{\epsilon} .
$$

The spreading code sequence is assigned to each users randomly:

$$
P(\mathbf{H})=\frac{1}{2^{N K}} .
$$

Let us here discuss the meaning of the spreading code $\mathbf{H}$. If one does not contrive any ways to demodulate the signals of users, the digital signals become mixed (interfere) with each signal, and then one cannot demodulate the original information at base station. We therefore divide the signal interval into $K$ chip intervals, where the interval is called pitch. Assigning such a spreading code to each user preliminarily, a base station catches the signal containing a noise. Thus, the problem is to estimate the sequence, $\sigma=\left(\sigma_{1}, \ldots, \sigma_{N}\right)$, which yields a satisfactory candidate for the original bit sequence $\xi$ from the received sequence $\boldsymbol{y}$. Then, the probability distribution of received information is written as

$$
P(\boldsymbol{y} \mid \boldsymbol{\sigma})=\left(\sqrt{\frac{\beta}{2 \pi}}\right)^{K} \exp \left(-\frac{\beta}{2}\left\|\boldsymbol{y}-\frac{\mathbf{H} \boldsymbol{\sigma}}{\sqrt{N}}\right\|^{2}\right)
$$

where $\beta=1 / T$ corresponds to inverse temperature in terms of statistical mechanics assuming that channel noise is generated from an additive white Gaussian. In other words, the temperature corresponds to the controlled noise power. If it approaches the true noise power, the performance of demodulation is supposed to get better.

By using the Bayes formula,

$$
P(A \mid B)=\frac{P(B \mid A) P(A)}{\sum_{A} P(B \mid A) P(A)},
$$

the posterior is described as a canonical distribution with Hamiltonian $H(\sigma)$ as

$$
\begin{gathered}
P(\boldsymbol{\sigma} \mid \boldsymbol{y})=\frac{\exp [-\beta H(\boldsymbol{\sigma})]}{Z}, \\
Z=\operatorname{Tr}_{\boldsymbol{\sigma}} \exp [-\beta H(\boldsymbol{\sigma})], \\
H(\boldsymbol{\sigma})=\frac{1}{2 N} \sum_{i, j} \sum_{k=1}^{K} \eta_{i}^{k} \eta_{j}^{k} \sigma_{i} \sigma_{j}-\frac{1}{\sqrt{N}} \sum_{i=1}^{N} \sum_{k=1}^{K} \eta_{i}^{k} y^{k} \sigma_{i},
\end{gathered}
$$


where we use the condition in Eq. (6) and the priors:

$$
P(\sigma)=\frac{1}{2^{N}},
$$

which means that both the spreading code and the original information follow uniform distributions. The Hamiltonian (10) is exactly the same as the so-called "anti-Hopfield model" with a random field on each neuron $\sigma_{i}$ regarding $\sigma$ as a neuronal state. It should be noted that the problem of estimating $\boldsymbol{y}$ from $\boldsymbol{\xi}$ is closely related to that of CS. The problem actually becomes identical to CS with $l_{p}$ norm by assuming $P\left(\sigma_{i}\right) \propto$ $\exp \left(-\beta\left|\sigma_{i}\right|^{p}\right)$ in the limit of $\beta \rightarrow \infty$ instead of Eq. (11) as a prior [17].

We next introduce the maximizer of the a posteriori marginal (MPM) estimate, i.e., finite temperature demodulation. We compare the two probabilities that $\sigma_{i}$ takes, 1 or -1 , for a given $\boldsymbol{y}$, viz, $P\left(\sigma_{i}= \pm 1 \mid \boldsymbol{y}\right)$, to construct the estimate and follow the decision of the "majority group." Hence, the MPM estimate for each bit is now given by

$$
\begin{aligned}
\tilde{\sigma}_{i} & =\operatorname{sgn}\left[P\left(\sigma_{i}=1 \mid \boldsymbol{y}\right)-P\left(\sigma_{i}=-1 \mid \boldsymbol{y}\right)\right] \\
& =\operatorname{sgn}\left[\sum_{\sigma_{i}= \pm 1} \sigma_{i} P\left(\sigma_{i} \mid \boldsymbol{y}\right)\right] \\
& =\operatorname{sgn}\left\langle\sigma_{i}\right\rangle_{\beta},
\end{aligned}
$$

where we defined the marginal:

$$
P\left(\sigma_{i} \mid \boldsymbol{y}\right)=\sum_{\boldsymbol{\sigma} \neq \sigma_{i}} P(\boldsymbol{\sigma} \mid \boldsymbol{y}),
$$

where $\langle\cdot\rangle$ indicates the thermal average.

The maximum a posteriori (MAP) estimate, on the other hand, corresponds to searching the ground state of (10). Therefore, the MAP estimate can be recovered from the MPM estimate in the zero temperature limit $T=0$.

We introduce an overlap between original signal $\boldsymbol{\xi}$ and estimated bit $\operatorname{sgn}\left\langle\sigma_{i}\right\rangle_{\beta}$ to investigate the average-case performance of the demodulation, viz.

$$
M(\boldsymbol{y}, \boldsymbol{\xi} \mid \mathbf{H})=\frac{1}{N} \sum_{i} \xi_{i} \operatorname{sgn}\left\langle\sigma_{i}\right\rangle_{\beta} .
$$

The above quantity is expected to be "self-averaging" in the limit of $N \rightarrow \infty$. This means that observables such as $M(\boldsymbol{y}, \boldsymbol{\xi} \mid \mathbf{H})$ for a given realization of the data set, $\boldsymbol{y}, \boldsymbol{\xi}$ and $\mathbf{H}$, become identical to the average of itself over the distribution of the Gaussian channel and spreading code, viz.,

$$
\begin{aligned}
\lim _{N \rightarrow \infty} M(\boldsymbol{y}, \boldsymbol{\xi} \mid \mathbf{H}) & =M(\beta) \\
& \equiv \sum_{\mathbf{H}} \operatorname{Tr}_{\xi} \int d \boldsymbol{y} P(\mathbf{H}) P(\boldsymbol{\xi}) P(\boldsymbol{y} \mid \boldsymbol{\xi}) \xi_{i} \operatorname{sgn}\left\langle\sigma_{i}\right\rangle_{\beta} \\
& =\left[\xi_{i} \operatorname{sgn}\left\langle\sigma_{i}\right\rangle_{\beta}\right],
\end{aligned}
$$

where the brackets [.] stand for the average over the data distribution $P(\mathbf{H}) P(\boldsymbol{\xi}) P(\boldsymbol{y} \mid \boldsymbol{\xi})$. Hence, $M(\beta)$ is apparently a suitable measurement for the average-case performance of the CDMA system.

For evaluating the average-case performance by the overlap, we need to know the information of true noise and true message preliminary. Thus, we assume the probabilistic distribution of them as prior.
We first assume that true noise $\epsilon$ in Eq. (1) follows an additive Gaussian channel with mean zero and $\sigma_{0}$ variance to explicitly calculate the above $M$ :

$$
\begin{aligned}
P(\boldsymbol{\epsilon}) & =\left(\frac{1}{\sqrt{2 \pi \sigma_{0}^{2}}}\right)^{2} \exp \left(-\frac{1}{2 \sigma_{0}^{2}}\|\boldsymbol{\epsilon}\|^{2}\right) \\
& =\left(\frac{1}{\sqrt{2 \pi \sigma_{0}^{2}}}\right)^{2} \exp \left(-\frac{1}{2 \sigma_{0}^{2}}\left\|\boldsymbol{y}-\frac{\mathbf{H} \boldsymbol{\xi}}{\sqrt{N}}\right\|^{2}\right) .
\end{aligned}
$$

For simplicity, we also assume that the information symbols are independent and identically distributed random variables:

$$
P(\xi)=\prod_{i=1}^{N} P\left(\xi_{i}\right)=\frac{1}{2^{N}} .
$$

Hence, as the temperature in Eq. (6) corresponds to the controlling parameter of the communication channel in the literature on the MPM estimate, optimal performance should be achieved under the condition $\beta=1 / T=\beta_{0}=1 / T_{0}=1 / \sigma_{0}^{2}$, which is nothing but the so-called Nishimori temperature.

Although the above formulation is given for the CDMA model based on Bayesian statistics, we will extend it to the quantum-mechanical version by simply adding the transverse field as

$$
\begin{gathered}
\hat{H}=\hat{H}_{0}+\hat{H}_{1}, \\
\hat{H}_{0}=\frac{1}{2 N} \sum_{i, j} \sum_{k=1}^{K} \eta_{i}^{k} \eta_{j}^{k} \hat{\sigma}_{i}^{z} \hat{\sigma}_{j}^{z}-\frac{1}{\sqrt{N}} \sum_{i=1}^{N} \sum_{k=1}^{K} \eta_{i}^{k} y^{k} \hat{\sigma}_{i}^{z}, \\
\hat{H}_{1}=-\Gamma \sum_{i=1}^{N} \hat{\sigma}_{i}^{x},
\end{gathered}
$$

where $\hat{\sigma}_{i}^{x}$ and $\hat{\sigma}_{i}^{z}$ denote the $x$ and $y$ components of the Pauli matrix, and $H_{1}$ is the transverse field causing quantum tunneling. The strength of the transverse field can be controlled by $\Gamma$.

Let us consider a single-spin system to intuitively figure out the quantum effect in the last equation. Denoting the eigenstates of $\hat{\sigma}^{z}$ as $|+\rangle=(1,0)^{t}$ and $|-\rangle=(0,1)^{t}$, the $x$ component of the Pauli matrix becomes $\hat{\sigma}^{x}=|+\rangle\langle-|+|-\rangle\langle+|$. Taking into account relation $\hat{\sigma}^{x}| \pm\rangle=|\mp\rangle$, we find that up-state $|+\rangle$ transits to down-state $|-\rangle$ and vice versa. This means that the transverse field induces the transitions between states by means of tunneling. The Ising spins in the Hamiltonian (10) are quantized as Pauli matrices in the framework (21). The transverse field is introduced into the Hamiltonian as a noncommutative term. Here, we should bear in mind that the key point of the QMPM estimate is to generate an appropriate ensemble that "imitates" the actual noise of the Gaussian channel by making use of thermal and quantum fluctuations.

The estimated bit in terms of the QMPM estimate that corresponds to (14) can be written as

$$
\begin{aligned}
\tilde{\sigma}_{i} & =\operatorname{sgn}\left[\operatorname{Tr}_{s}\left(\hat{\sigma}_{i}^{z} \hat{\rho}\right)\right] \\
& =\operatorname{sgn}\left(\left\langle\hat{\sigma}_{i}^{z}\right\rangle_{\beta, \Gamma}\right),
\end{aligned}
$$


where $\hat{\rho} \equiv e^{-\beta \hat{H}} / \operatorname{Tr} e^{-\beta \hat{H}}$. Consequently, the overlap for the case of the quantum system (21) is evaluated as

$$
\begin{aligned}
M(\beta, \Gamma) & =\sum_{\mathbf{H}} \operatorname{Tr}_{\xi} \int d \boldsymbol{y} P(\mathbf{H}) P(\boldsymbol{\xi}) P(\boldsymbol{y} \mid \boldsymbol{\xi}) \xi_{i} \operatorname{sgn}\left(\left\langle\hat{\sigma}_{i}^{z}\right\rangle_{\beta, \Gamma}\right) \\
& =\left[\xi_{i} \operatorname{sgn}\left(\left\langle\hat{\sigma}_{i}^{z}\right\rangle_{\beta, \Gamma}\right)\right]
\end{aligned}
$$

Note that the overlap for QMPM depends on the strength of thermal fluctuations controlled by the inverse temperature $\beta$, and the amplitude of quantum fluctuations determined by the strength of the transverse field $\Gamma$. The true noise power $\beta_{0}=$ $1 / \sigma_{0}^{2}$ in $P(\boldsymbol{y} \mid \boldsymbol{\xi})$ is given in this study because of investigating the demodulation performance, in particular, the overlap as a function of $\beta$ around the true value $\beta_{0}$.

\section{ANALYSIS}

We derive saddle point equations that determine the equilibrium state by using the standard replica method to explicitly evaluate performance through the QMPM estimate.

We consider the limit, $N, K \rightarrow \infty$, to analyze the multiuser demodulation problem while retaining the ratio,

$$
\alpha=\frac{K}{N},
$$

of the order 1 object. We apply Suzuki-Trotter (ST) decomposition [18],

$$
\exp \left(\hat{H}_{0}+\hat{H}_{1}\right)=\lim _{P \rightarrow \infty}\left(e^{\hat{H}_{0} / P} e^{\hat{H}_{1} / P}\right)^{P},
$$

to the partition function, $Z=\operatorname{Tr} \exp (-\beta \hat{H})$, with Eq. (21) to cast the problem as an equivalent classical spin system to achieve our goal. As a result, the partition function $Z$ and the effective Hamiltonian $H_{\text {eff }}$ are given by

$$
\begin{aligned}
Z=\lim _{P \rightarrow \infty}\left(\frac{1}{2} \sinh \frac{2 \beta \Gamma}{P}\right)^{N P / 2} \operatorname{Tr}_{\{\sigma(t)\}} \exp \left(-H_{\mathrm{eff}}\right), \\
H_{\mathrm{eff}}=\frac{\beta}{2 N P} \sum_{t=1}^{P} \sum_{i, j} \sum_{k=1}^{K} \eta_{i}^{k} \eta_{j}^{k} \sigma_{i}(t) \sigma_{j}(t) \\
-\frac{\beta}{\sqrt{N} P} \sum_{t=1}^{P} \sum_{i=1}^{N} \sum_{k=1}^{K} \eta_{i}^{k} y^{k} \sigma_{i}(t) \\
-\frac{1}{2} \ln \frac{\beta \Gamma}{P} \sum_{t=1}^{P} \sum_{i=1}^{N} \sigma_{i}(t) \sigma_{i}(t+1),
\end{aligned}
$$

where $P$ is called the Trotter number and $t$ is the Trotter index. The symbol $\operatorname{Tr}_{\{\sigma(t)\}}$ denotes the trace over the $\sigma(t)$ spins. With attention to the above equation, the quantum system with the transverse field is described as the classical system on the space which has an extra dimension. The coupled term between Trotter indices in Eq. (31) emerges because of manipulation for diagonalization of the transverse field term $\Gamma \sum_{i} \hat{\sigma}^{x}$ [18].

Using the well-known replica method,

$$
[\ln Z]=\lim _{n \rightarrow 0} \frac{\left[Z^{n}\right]-1}{n},
$$

we calculate the averaged free energy density, $[\ln Z]$, in terms of $\left[Z^{n}\right]$. The replicated partition function is now written as

$$
\begin{aligned}
{\left[Z^{n}\right]=} & \sum_{\mathbf{H}} P(\mathbf{H}) \underset{\xi}{\operatorname{Tr}} P(\boldsymbol{\xi}) \int \prod_{k} d y^{k} P\left(y^{k} \mid \boldsymbol{\xi}\right) \underset{\left\{\boldsymbol{\sigma}^{\mu}(t)\right\}}{\operatorname{Tr}} \exp \left(-\sum_{\left\{\boldsymbol{\sigma}^{\mu}(t)\right\}} H_{\mathrm{eff}}\left(\boldsymbol{\sigma}^{\mu}(t)\right)\right) \\
= & \frac{1}{2^{N}} \frac{1}{2^{N K}} \sum_{\mathbf{H}} \operatorname{Tr}_{\xi} \int \prod_{k} d y^{k}\left(\frac{\beta_{0}}{2 \pi}\right)^{1 / 2} \exp \left(-\frac{\beta_{0}}{2}\left(y^{k}-\frac{1}{\sqrt{N}} \sum_{i} \eta_{i}^{k} \xi_{i}\right)^{2}\right) \\
& \times \operatorname{Tr}_{\left\{\boldsymbol{\sigma}^{\mu}(t)\right\}} \exp \left(-\frac{\beta}{2 N P} \sum_{t, \mu} \sum_{i, j} \sum_{k} \eta_{i}^{k} \eta_{j}^{k} \sigma_{i}^{\mu}(t) \sigma_{j}^{\mu}(t)+\frac{\beta}{\sqrt{N} P} \sum_{t, \mu} \sum_{i} \sum_{k} \eta_{i}^{k} y^{k} \sigma_{i}^{\mu}(t)+B \sum_{t, \mu} \sum_{i} \sigma_{i}^{\mu}(t) \sigma_{i}^{\mu}(t+1)\right),
\end{aligned}
$$

where $B \equiv \frac{1}{2} \ln \operatorname{coth} \frac{\beta \Gamma}{P},\left\{\sigma^{\mu}(t)\right\}=\left[\sigma^{1}(t), \ldots, \sigma^{n}(t)\right]$, and $t=1, \ldots, P$. The replica indices are denoted by $\mu$. Introducing the following order parameters:

$$
\begin{aligned}
R_{\mu}(t) & =\frac{1}{N} \sum_{i} \xi_{i} \sigma_{i}^{\mu}(t), \\
Q_{\mu \nu}\left(t, t^{\prime}\right) & =\frac{1}{N} \sum_{i} \sigma_{i}^{\mu}(t) \sigma_{i}^{\nu}\left(t^{\prime}\right), \\
Q_{\mu \mu}\left(t, t^{\prime}\right) & =\frac{1}{N} \sum_{i} \sigma_{i}^{\mu}(t) \sigma_{i}^{\mu}\left(t^{\prime}\right),
\end{aligned}
$$

with $Q_{\mu \mu}(t, t)=1$, the free energy density $f$ is given by

$$
\begin{aligned}
-\beta f= & \frac{\alpha}{2}\left\{-\ln [1-\beta(q-\chi)]+\frac{\beta\left(1+\beta_{0}\right)}{\beta_{0}}+\frac{\beta\left[2 R-q-\left(1+\beta_{0}^{-1}\right)\right]}{1-\beta(q-\chi)}+\beta(\chi-1)\right\}-\hat{R} R \\
& -\hat{\chi} \chi+\frac{\hat{q} q}{2}+\int D z \ln \left(\int D w 2 \cosh \sqrt{\Phi^{2}+\beta^{2} \Gamma^{2}}\right),
\end{aligned}
$$




$$
\Phi=\frac{\phi}{\beta}=z \sqrt{2 \hat{\chi}-\hat{q}}+w \sqrt{\hat{q}}+\hat{R},
$$

under replica symmetry (RS) and static approximation (SA), viz., $R_{\mu}(t)=R, Q_{\mu \nu}\left(t, t^{\prime}\right)=q$, and $Q_{\mu \mu}\left(t, t^{\prime}\right)=\chi\left(t \neq t^{\prime}\right)$ in the limit of $N \rightarrow \infty$. $\hat{R}, \hat{q}$, and $\hat{\chi}$ correspond to conjugate Lagrange multipliers of $R, q$, and $\chi$. The SA means that the order parameter does not depend on the Trotter dimension [19]. For this manipulation, we can derive the free energy without imaginary time by the standard replica method. The saddle point equations are given by

$$
\begin{gathered}
R=\int D w \int D z \frac{\Phi \sinh \Xi}{\Omega \Xi} \\
q=\int D w\left(\int D z \frac{\Phi \sinh \Xi}{\Omega \Xi}\right)^{2} \\
\chi=\int \frac{D w}{\Omega} \int D z\left(\frac{\beta^{2} \Gamma^{2} \sinh \Xi}{\Xi^{3}}+\frac{\Phi^{2} \cosh \Xi}{\Xi^{2}}\right), \\
\hat{R}=\frac{\alpha \beta}{1+\beta(\chi-q)}, \\
\hat{q}=\frac{\alpha \beta^{2}\left(1+q-2 R+\beta_{0}^{-1}\right)}{[1+\beta(\chi-q)]^{2}} \\
2 \hat{\chi}-\hat{q}=\frac{\alpha \beta^{2}(\chi-q)}{1+\beta(\chi-q)}
\end{gathered}
$$

$$
\begin{aligned}
& \Xi=\sqrt{\Phi^{2}+\beta^{2} \Gamma^{2}}, \\
& \Omega=\int D z \cosh \Xi,
\end{aligned}
$$

where $\int D u(\cdot)=\int_{-\infty}^{\infty} d u(\cdot) e^{-u^{2} / 2} / \sqrt{2 \pi}$. Considering the classical case of $\Gamma=0$, we easily find that these equations become identical to the classical version with $\chi=1$. The detailed calculations to derive the free energy density (38) are given in our Appendix. By comparing these expressions and Eqs. (40) and (41), we immediately find that $\int D z \frac{\Phi \sinh \Xi}{\Omega \Xi}$ is closely related to $\left\langle\hat{\sigma}_{i}\right\rangle_{\beta, \Gamma}$. Thus, the final form of the overlap as a performance measurement is easily obtained as [8]

$$
M(\beta, \Gamma)=\int D w \operatorname{sgn}\left(\int D z \frac{\Phi \sinh \Xi}{\Omega \Xi}\right) .
$$

It should be noted that the overlap for classical MPM is recovered by setting $\Gamma=0$ in the above expression (48).

\section{RESULTS}

We evaluate the performance of demodulation with quantum and thermal fluctuations by numerically solving Eqs. (40)(48) in the following.

\section{A. Upper bound of overlap}

We first derive the inequality for the overlap to clarify the upper bound. The overlap in the classical case defined by Eq. (17) can be written as follows:

$$
\begin{aligned}
M(\beta) & =\underset{\xi}{\operatorname{Tr}} \int \prod_{k} d y^{k} C_{N K} \exp \left(-\frac{\beta_{0}}{2}\left(y^{k}\right)^{2}\right) \exp \left(-\frac{\beta_{0}}{2 N} \sum_{i, j} \eta_{i}^{k} \eta_{j}^{k} \xi_{i} \xi_{j}+\frac{\beta_{0}}{\sqrt{N}} \sum_{i} \xi_{i} \eta_{i} y^{k}\right) \xi_{i} \operatorname{sgn}\left(\frac{\operatorname{Tr} \sigma_{i} e^{-\beta H}}{\operatorname{Tr} e^{-\beta H}}\right) \\
& \leqslant \int \prod_{k} d y^{k} C_{N K} \exp \left(-\frac{\beta_{0}}{2}\left(y^{k}\right)^{2}\right)\left\|\operatorname{Tr}_{\xi} \xi_{i} \exp \left(-\frac{\beta_{0}}{2 N} \sum_{i, j} \eta_{i}^{k} \eta_{j}^{k} \xi_{i} \xi_{j}+\frac{\beta_{0}}{\sqrt{N}} \sum_{i} \xi_{i} \eta_{i} y^{k}\right)\right\|\left\|\operatorname{sgn}\left(\frac{\operatorname{Tr} \sigma_{i} e^{-\beta H}}{\operatorname{Tr} e^{-\beta H}}\right)\right\| \\
& \leqslant \int \prod_{k} d y^{k} C_{N K} \exp \left(-\frac{\beta_{0}}{2}\left(y^{k}\right)^{2}\right)\left\|\operatorname{Tr}_{\xi} \xi_{i} \exp \left(-\frac{\beta_{0}}{2 N} \sum_{i, j} \eta_{i}^{k} \eta_{j}^{k} \xi_{i} \xi_{j}+\frac{\beta_{0}}{\sqrt{N}} \sum_{i} \xi_{i} \eta_{i} y^{k}\right)\right\| \\
& \equiv M_{\max }^{\text {classic }} .
\end{aligned}
$$

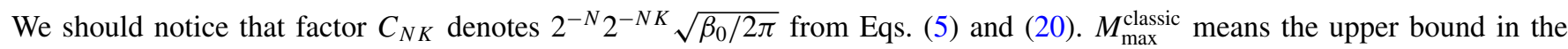
classical case. However, the overlap for the quantum case is given by

$$
\begin{aligned}
& M(\beta, \Gamma)=\operatorname{Tr}_{\xi} \int \prod_{k} d y^{k} C_{N K} \exp \left(-\frac{\beta_{0}}{2}\left(y^{k}\right)^{2}\right) \exp \left(-\frac{\beta_{0}}{2 N} \sum_{i, j} \eta_{i}^{k} \eta_{j}^{k} \xi_{i} \xi_{j}+\frac{\beta_{0}}{\sqrt{N}} \sum_{i} \xi_{i} \eta_{i} y^{k}\right) \xi_{i} \operatorname{sgn}\left(\frac{\operatorname{Tr} \hat{\sigma}_{i}^{z} e^{-\beta \hat{H}}}{\operatorname{Tr} e^{-\beta \hat{H}}}\right) \\
& \leqslant \int \prod_{k} d y^{k} C_{N K} \exp \left(-\frac{\beta_{0}}{2}\left(y^{k}\right)^{2}\right)\left\|\operatorname{Tr}_{\xi} \xi_{i} \exp \left(-\frac{\beta_{0}}{2 N} \sum_{i, j} \eta_{i}^{k} \eta_{j}^{k} \xi_{i} \xi_{j}+\frac{\beta_{0}}{\sqrt{N}} \sum_{i} \xi_{i} \eta_{i} y^{k}\right)\right\|\left\|\operatorname{sgn}\left(\frac{\operatorname{Tr} \hat{\sigma}_{i}^{z} e^{-\beta \hat{H}}}{\operatorname{Tr} e^{-\beta \hat{H}}}\right)\right\| \\
& \leqslant \int \prod_{k} d y^{k} C_{N K} \exp \left(-\frac{\beta_{0}}{2}\left(y^{k}\right)^{2}\right)\left\|\underset{\xi}{\operatorname{Tr}} \xi_{i} \exp \left(-\frac{\beta_{0}}{2 N} \sum_{i, j} \eta_{i}^{k} \eta_{j}^{k} \xi_{i} \xi_{j}+\frac{\beta_{0}}{\sqrt{N}} \sum_{i} \xi_{i} \eta_{i} y^{k}\right)\right\| \\
& \equiv M_{\max }^{\text {classic }} \text {. }
\end{aligned}
$$


(a)

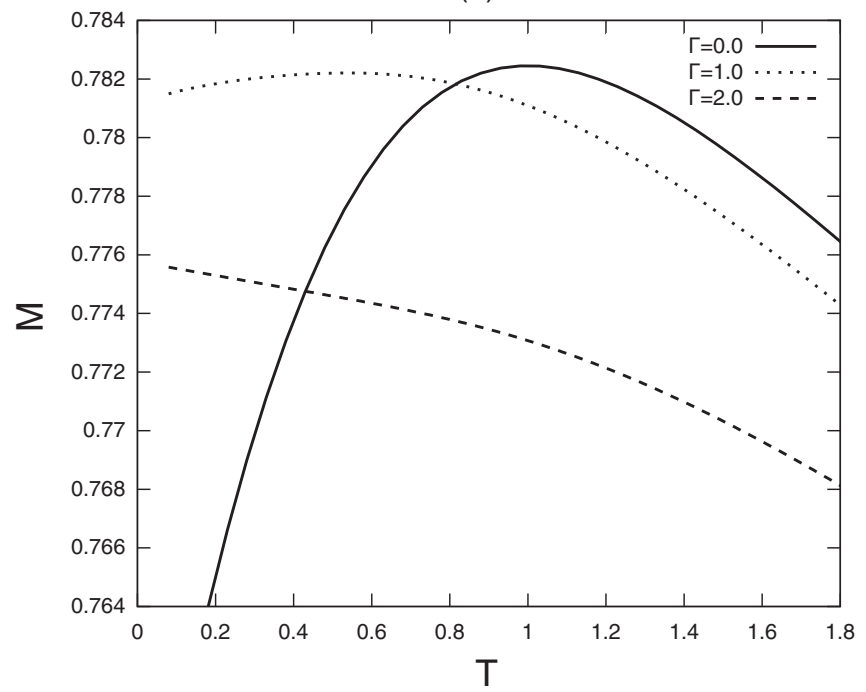

(b)

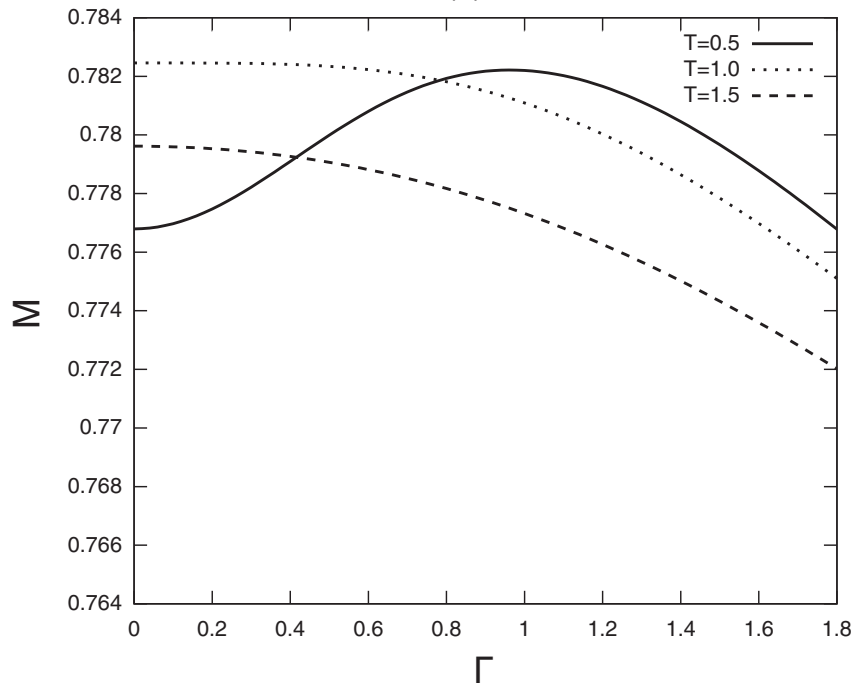

FIG. 2. (a) Dependence of overlap $M$ on level of thermal fluctuations $T$ for $\alpha=2.0$ and $T_{0}=1.0$. (b) Dependence of overlap $M$ on level of quantum fluctuations $\Gamma$ for $\alpha=2.0$ and $T_{0}=1.0$.

Thus, the optimal demodulating performance of the MPM estimate in the presence of quantum fluctuations is the same as that in the thermal fluctuations. We can confirm that overlap is maximum at the Nishimori temperature, $T_{0}=1 / \beta_{0}$, as

$$
\begin{aligned}
M_{\max }^{\text {classic }} & =\operatorname{Tr}_{\xi} \int \prod_{k} d y^{k} C_{N K} \exp \left(-\frac{\beta_{0}}{2}\left(y^{k}-\frac{1}{\sqrt{N}} \sum_{i} \eta_{i}^{k} \xi_{i}\right)^{2}\right) \xi_{i} \frac{\operatorname{Tr}_{\xi} \xi_{i} \exp \left(-\frac{\beta_{0}}{2 N} \sum_{i, j} \eta_{i}^{k} \eta_{j}^{k} \xi_{i} \xi_{j}+\frac{\beta_{0}}{\left.\sqrt{N} \sum_{i} \xi_{i} \eta_{i} y^{k}\right)}\right.}{\| \operatorname{Tr}_{\xi} \xi_{i} \exp \left(-\frac{\beta_{0}}{2 N} \sum_{i, j} \eta_{i}^{k} \eta_{j}^{k} \xi_{i} \xi_{j}+\frac{\beta_{0}}{\left.\sqrt{N} \sum_{i} \xi_{i} \eta_{i} y^{k}\right) \|}\right.} \\
& =\underset{\xi}{\operatorname{Tr}} \int \prod_{k} d y^{k} C_{N K} \exp \left(-\frac{\beta_{0}}{2}\left(y^{k}-\frac{1}{\sqrt{N}} \sum_{i} \eta_{i}^{k} \xi_{i}\right)^{2}\right) \xi_{i} \operatorname{sgn}\left\langle\xi_{i}\right\rangle_{\beta_{0}}=M\left(\beta_{0}\right) .
\end{aligned}
$$

The inequality means that the optimal demodulating performance through the MPM estimate incorporating the transverse field cannot exceed the optimal one in the classical case. However, we must mention that as the $\Gamma, T$ dependence of demodulating performance on the QMPM estimate could not be clarified with the above argument, we next need to numerically solve the saddle point equations (40)-(48) to make the issue clearer.

\section{B. Behavior around peak}

Figure 2 plots the dependence of overlap $M$ on $T$ and $\Gamma$ for the case of $\alpha=2.0$ and $T_{0}=1.0$. We find that the overlap has a single peak at $T=T_{0}=1.0$ for the case without transverse field $\Gamma=0$ as is well known [see Fig. 2(a)]. This means that the optimal performance in demodulation is achieved at some temperature $T=T_{0}$ that corresponds to the true variance in the Gaussian channel described as Eq. (19). Also note that the overlap appropriately exhibits a peak that is obtained by controlling the strength of the quantum fluctuation, $\Gamma$. The height of the peak seems to be the same as that of the CMPM estimate. These results are consistent with those in our previous studies [7,8].

We next investigate the overlap from the viewpoint of the $\Gamma-T$ diagram in Fig. 3(a). The gradation indicates the values of the overlap and the solid line represents the peaks of the overlap, $M_{\text {top. }}$. We can observe that $M_{\text {top }}$ exists in some range of temperature below the Nishimori temperature $T=T_{0}$. We should keep in mind that the numerical solution to the overlap in the quite low temperature region $(T<0.05)$ cannot be obtained within reliable precision due to limitations in our computational resources. We can see the dependence of $M_{\text {top }}$ on $\Gamma$ that is indicated by the solid line in Fig. 3(b) for $\alpha=2.0$ and $T_{0}=1.0$. We find that the peak of overlap $M_{\text {top }}$ decreases monotonically from 0.7824 to 0.7816 . Therefore, we must conclude that quantum fluctuations worsen optimal performance slightly for large $\Gamma$. Obviously, the results are consistent with our argument using the inequalities (49)-(51).

\section{Dependence of demodulating performance on chip ratio}

The dependence of overlap on $\alpha$ is plotted in Figs. 4(a) ( $\left.T_{0}=1.0, T=1.0\right)$ and (b) $\left(T_{0}=0.08, T=0.08\right)$ for various $\Gamma$ values. As the temperature is set to the Nishimori temperature for both cases, the overlap has a peak at $\Gamma=0$ as we noted in the previous section. Since parameter $\alpha$ means the chip ratio, we naturally assumed that overlap would increase as $\alpha$ increased. We find that the slope of increase in the overlap for the quantum case is much gentler than that for the classical case.

When the variance of the Gaussian channel $T_{0}$ is very small, the first-order phase transition takes place around $\alpha \simeq 0.6$ for 
(a)

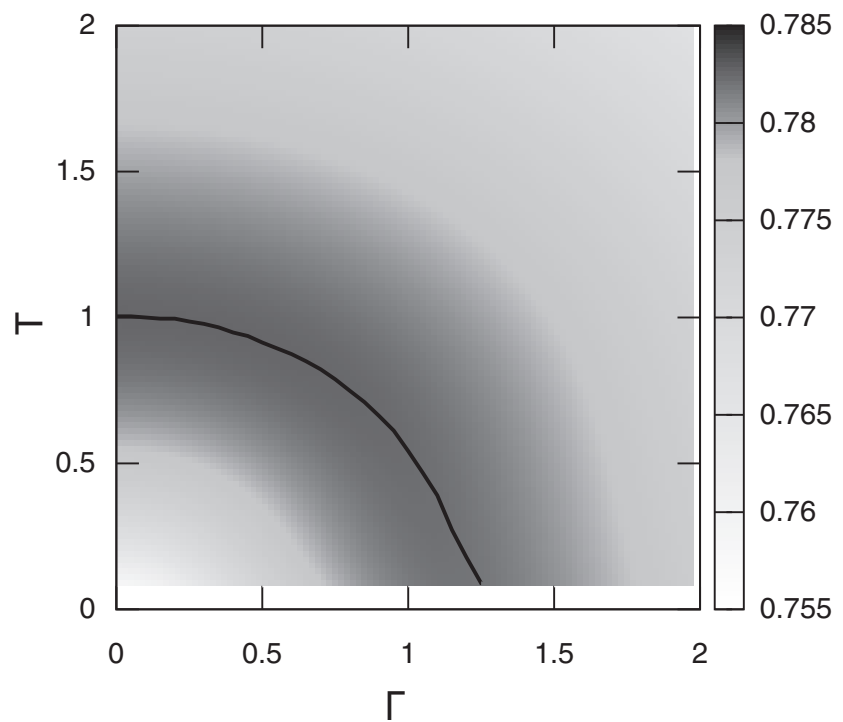

(b)

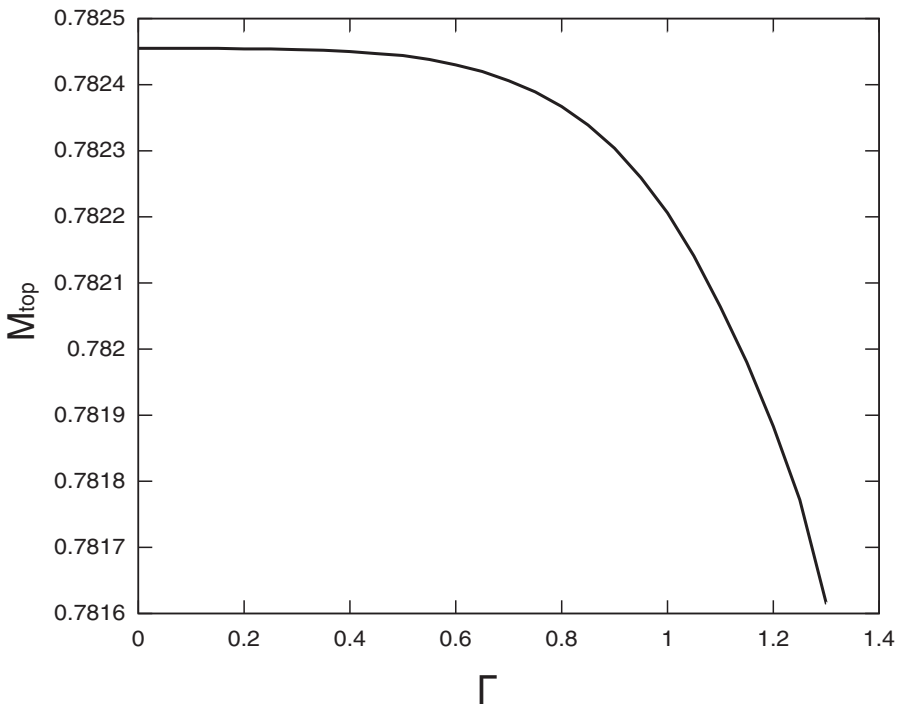

FIG. 3. (a) Value of overlap on $\Gamma-T$ plane for $T_{0}=1.0$ and $\alpha=2.0$. Solid line indicates location of peak in overlap. (b) Dependence of $M_{\text {top }}$ on $\Gamma$ corresponding to solid line in panel (a).

$\Gamma=0$. In contrast, we find that for relatively large quantum fluctuations, $\Gamma=0.5$, the overlap continuously converges to unity. These results imply that quantum fluctuations never improve the average-case performance of MPM estimate for any choice of the chip ratio $\alpha$.

We confirm that two possible solutions coexist at low temperature [see the inset of Fig. 4(b)], which lead to a sort of hysteresis phenomenon. Such a bistable region obtained under the RS and SA ansatz disappears as $\Gamma$ increases. The spinodal lines in $(\alpha, \Gamma)$ space are plotted in Fig. 5. The distinction between the solid and dashed lines comes from the dependence of $\Gamma$ on $\alpha_{1}$ (solid) or $\alpha_{2}$ (dashed). As can be shown in Fig. 5(a) $T_{0}=T=0.08$, the region of $\alpha$ in which solutions coexist is maximum at $\Gamma=0$. However, there is also a coexistence

(a)

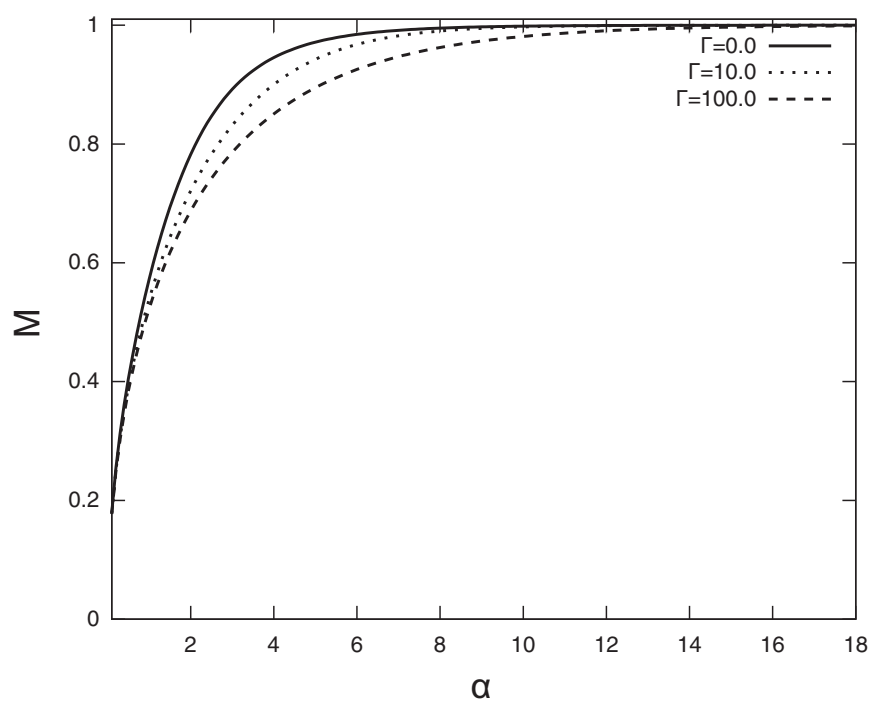

region for solutions to $T_{0}=0.08$ and $T=0.05$ with slightly different shapes from those of $T_{0}=T=0.08$. The coexistence region gradually narrows for the both cases as $\Gamma$ increases, and the region eventually disappears. Similar behavior has been found in $(\alpha, T)$ space when $T_{0}$ is fixed for the classical case, i.e., $\Gamma=0[20]$.

\section{Improvable region for demodulating performance}

The previous section explained our investigation into the average-case performance of the demodulation by means of the QMPM estimate. We focused on the location of the peak of the overlap. Here, we will discuss the conditions under which the performance of the QMPM estimate is better than

(b)

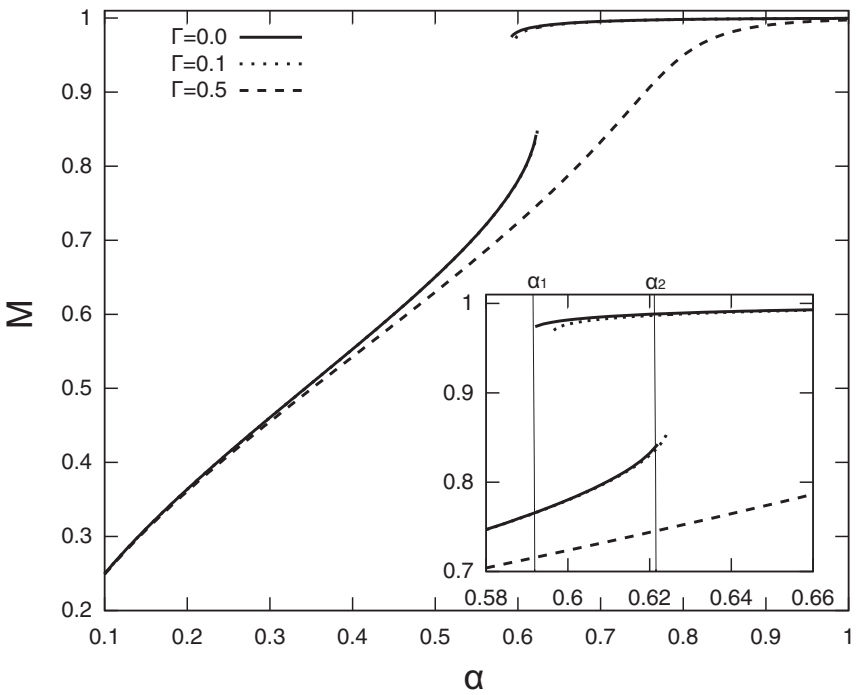

FIG. 4. Overlap $M$ vs chip ratio $\alpha$. (a) Results for $T_{0}=1.0$ and $T=T_{0}$. (b) Results for $T_{0}=0.08$ and $T=T_{0}$. Bistable solutions coexist for $\Gamma=0$ in region $\alpha_{1}<\alpha<\alpha_{2}$. 
(a)

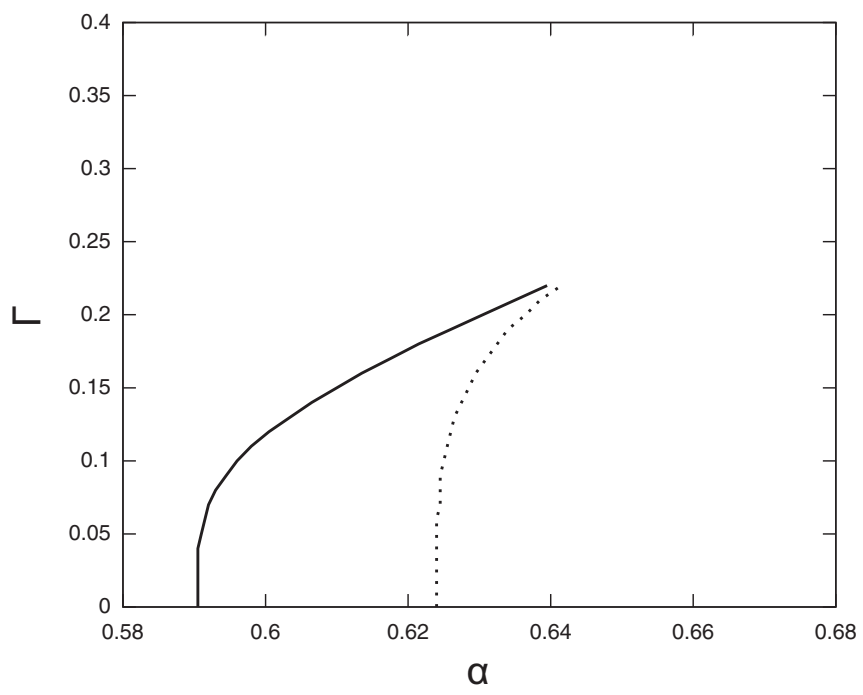

(b)

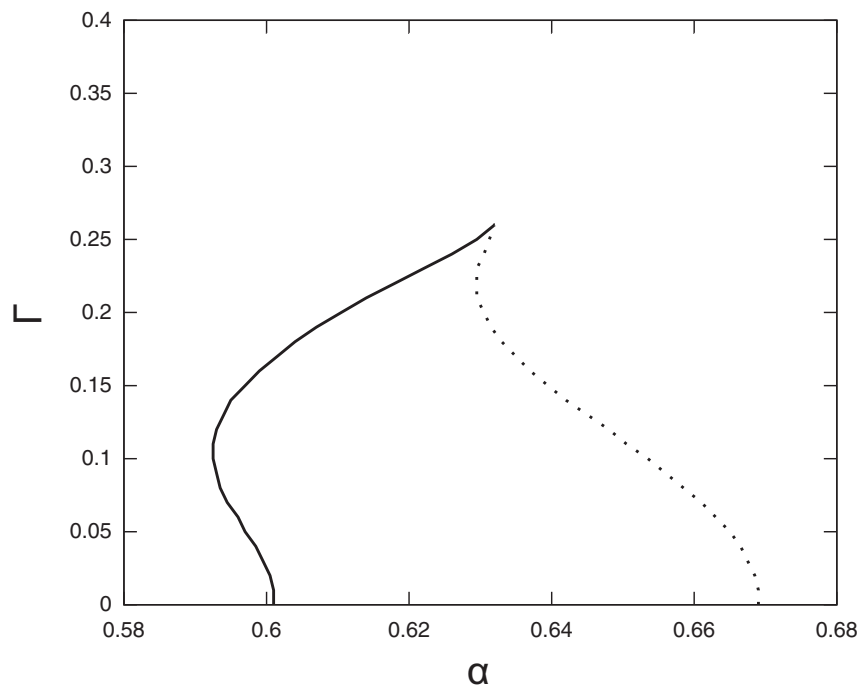

FIG. 5. (a) Spinodal line at $T_{0}=T=0.08$. (b) Spinodal line at $T_{0}=0.08$ and $T=0.05$. Solid line represents $\Gamma\left(\alpha_{1}\right)$ and dotted line represents $\Gamma\left(\alpha_{2}\right)$.

that of CMPM. This is a slightly different viewpoint from optimality in the overlap.

To quantify the degree of improvement achieved by quantum fluctuations, we introduce the following quantity:

$$
\Delta M(\Gamma, \Omega)=M(\Gamma, \Omega)-M(0, \Omega),
$$

i.e., the difference between the overlaps at $\Omega=\left\{T_{0}, T, \alpha\right\} \in$ $\mathcal{R}$ with and without the quantum fluctuations. The quality of quantum demodulation is better than that of classical demodulation for $\Delta M(\Gamma, \Omega)>0$. It should be noted that the overlap is maximized at $\Gamma=0$ for $T=T_{0}$, viz, $\Delta M\left(\Gamma, \Omega_{0}\right)<$ 0 for $\Omega_{0}=\left\{T_{0}, T=T_{0}, \alpha\right\} \in \mathcal{R}$ and $\Gamma>0 . \Delta M$ is always less than zero for the case of $T>T_{0}$, as we mentioned in the previous section. For these reasons, we define a region where

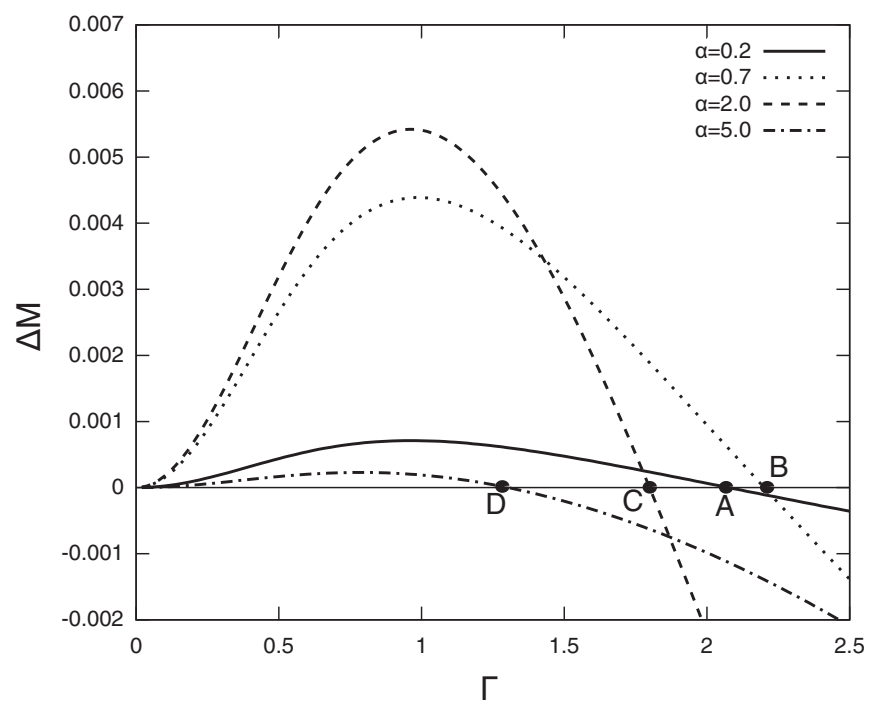

FIG. 6. Difference between overlaps with and without transverse field for $T_{0}=1.0$ and $T=0.5$. Closed circles labeled A, B, C, and $\mathrm{D}$ denote points at which $\Delta M=0$ holds.
$\Delta M>0$ an improvable region, whereas the region specified by $\Delta M<0$ is referred to as a worsened region for $T<T_{0}$.

We have plotted $\Delta M$ as a function of $\Gamma$ for $T_{0}=1.0$ and $T=0.5$ with various values of $\alpha$ in Fig. 6 . We find that $\Delta M$ has a peak in some range of $\Gamma$ and it eventually drops to a negative value. Demodulation achieves the best possible performance for a given set of $T$ and $T_{0}$ at some specific value of $\Gamma$ for which $\Delta M$ has a peak. We should note that $\Delta M=0$ determines the border of $\Gamma$ between improvable and worsened regions. We have marked the locations in which $\Delta M=0$ is satisfied for $\alpha=0.2,0.7,2.0$, and 0.5 with respective points labeled $\mathrm{A}, \mathrm{B}, \mathrm{C}$, and $\mathrm{D}$. The $\Gamma$ for $\Delta M=0$ is not a monotonic function of $\alpha$ because we clearly find that the inequality $\Gamma_{\mathrm{D}}<\Gamma_{\mathrm{C}}<\Gamma_{\mathrm{A}}<\Gamma_{\mathrm{B}}$ holds. This implies the existence of a suitable $\alpha$ to improve demodulating performance. Also note that the peaked value of $\Delta M$ decreases as $\alpha$ increases because both $M(\Gamma, \Omega)$ and $M(0, \Omega)$ converge to unity.

We will next investigate the critical $\Gamma(T)$ at which the improvable and worsened regions are clearly separated. The results are plotted in Figs. 7(a) and 7(b). The dashed lines were obtained under the conditions $\Delta M(\Gamma, \Omega)=0$. We find that the improvable region is extended up to the low temperature region in Fig. 7(a). Interestingly, the critical line (the $\Gamma-\alpha$ curve) that separates improvable and worsened regions has a nonmonotonic shape with a single maximum at some finite $\alpha$ value. This means that there is a suitable chip ratio $\alpha$ to improve demodulation performance.

\section{SUMMARY AND CONCLUDING REMARKS}

We investigated the average-case performance of a Bayesian CDMA multiuser detector that was extended by means of quantum fluctuations. The following three items summarize what we learned from this study.

1. Quantum fluctuations controlled by the transverse field could not improve the optimal performance of CMPM. To make matters worse, the MPM estimate that incorporated 
(a)

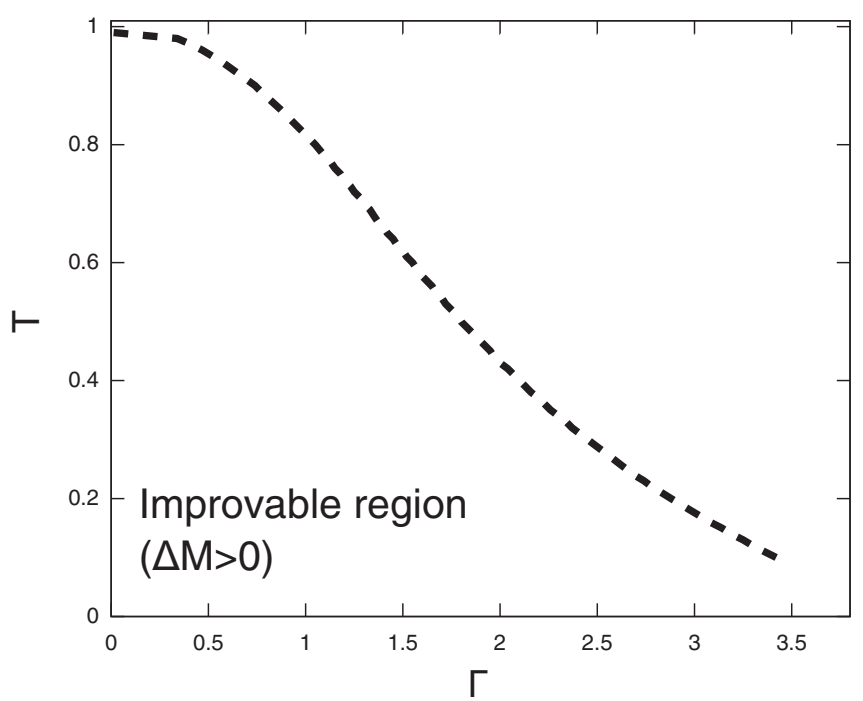

(b)

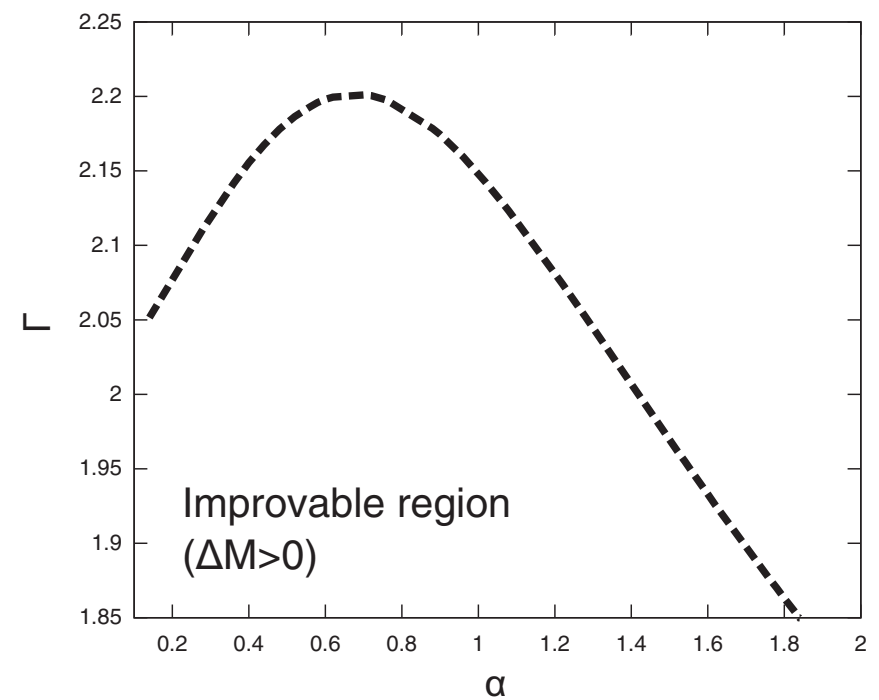

FIG. 7. Improvable regions in $T-\Gamma$ (left) and $\Gamma-\alpha$ (right) planes for $T_{0}=1.0$. Dashed lines indicate border at which $\Delta M=0$ holds for $\alpha=2.0$ (a) and $T=0.5$ (b).

the transverse field (QMPM estimate) never exhibited the same optimal performance as the conventional MPM estimate (CMPM estimate) even within the strictest sense. This conclusion was supported by a mathematically rigorous argument using inequality on the overlap.

2. There was an improvable region below the Nishimori temperature obtained by using the transverse field. Thus, the transverse field actually improved performance for some choices of nonoptimal parameters although optimal performance with the transverse field could not be improved.

3 . Increasing the chip ratio improved performance. The overlap actually eventually reached unity for both cases with and without the transverse field.

We should examine other problems, viz., image restoration and Sourlas code even though the performance of each was already extensively investigated $[7,8]$, to figure out what the universal properties of the transverse field were within the context of the MPM estimate in probabilistic information processing. The following discusses what is common or different behavior in these models.

\section{Image restoration}

Let us consider the process to restore a black and white image from degraded pixels $\boldsymbol{\tau}=\left\{\tau_{i}\right\}(i=1, \ldots, N)=\boldsymbol{\xi}+\boldsymbol{\epsilon}$ for original image $\xi=\left\{\xi_{i}\right\},\left(\xi_{i}= \pm 1, i=1, \ldots, N\right)$. Then, we accept the following two assumptions for the Markov random field (MRF) prior and Gaussian noise:

$$
\begin{gathered}
P(\xi) \propto \exp \left(\beta_{s} \sum_{\langle i, j\rangle} \xi_{i} \xi_{j}\right), \\
P(\boldsymbol{\tau} \mid \xi) \propto \exp \left\{-\frac{1}{2 a_{\tau}^{2}} \sum_{i}\left(\tau_{i}-\tau_{0} \xi_{i}\right)^{2}\right\},
\end{gathered}
$$

where $\beta_{s}=1 / T_{s}$ denotes the "true" smoothness among pixels and $a_{\tau}^{-2}$ is the true variance. The posterior distribution of estimated pixels is given for estimate $\sigma$ instead of true pixels $\xi$ as

$$
\begin{gathered}
P(\boldsymbol{\sigma} \mid \boldsymbol{\tau}) \propto \exp \left[-\beta H^{\mathrm{IR}}(\boldsymbol{\sigma})\right], \\
H_{0}^{\mathrm{IR}}(\boldsymbol{\sigma})=-\sum_{i, j} \sigma_{i} \sigma_{j}-h_{e} \sum_{i} \tau_{i} \sigma_{i},
\end{gathered}
$$

where $\beta=1 / T$ stands for the inverse temperature that corresponds to estimated smoothness and $h_{e}$ is the estimated noise power. The overlap is a measurement of restoration and it reads

$$
M\left(\beta, h_{e}\right)=\operatorname{Tr}_{\xi} \int d \boldsymbol{\tau} P(\xi) P(\boldsymbol{\tau} \mid \xi) \xi_{i} \operatorname{sgn}\left\langle\sigma_{i}\right\rangle_{\beta, h} .
$$

The overlap is maximized under the Nishimori condition as

$$
M\left(\beta, h_{e}\right) \leqslant M\left(\beta_{s}, \frac{\tau_{0}}{\beta_{s} a_{\tau}^{2}}\right) .
$$

Note that $h_{e}$ corresponds to $h / \beta$ in the conventional notation $[7,21]$.

\section{Sourlas code}

Sourlas code is now recognized in the community of physics as error-correcting codes that are described as a spin glass model with $p$-body interactions [10]. The basic idea of errorcorrecting codes is to add redundancy to the original messages so that receivers can recover the original message from a noisy redundant message. An original message is encoded as $p$ product bits $J_{i 1 \ldots i p}^{0}=\xi_{i 1} \ldots \xi_{i p}$ and then the received message can be represented as $\boldsymbol{J}=\boldsymbol{J}^{0}+\boldsymbol{\epsilon}$. The original message $\boldsymbol{\xi}$ is generated from a uniform distribution, i.e.,

$$
P(\xi)=\frac{1}{2^{N}},
$$


and the sender transmits all possible combinations ${ }_{N} C_{p}$ of the products of $p$ components in the $N$-dimensional vector $\xi$ through a Gaussian channel:

$P(\boldsymbol{J} \mid \boldsymbol{\xi}) \propto \exp \left\{-\frac{D_{N p}}{J^{2}} \sum_{i 1<\cdots<i p}\left(J_{i 1 \ldots i p}-\frac{J_{0}}{D_{N p}} \xi_{i 1} \ldots \xi_{i p}\right)\right\}$,

where $D_{N p}=N^{p-1} / p$ !. The sum $\sum_{i 1<\cdots<i p}$ runs over all possible combinations of $p$ spins out of $N$ spins. The posterior of the estimated sequence leads to

$$
\begin{gathered}
P(\boldsymbol{\sigma} \mid \boldsymbol{J}) \propto \exp \left(-\beta H_{0}^{\mathrm{EC}}\right), \\
H_{0}^{\mathrm{EC}}=-\sum_{i 1<\cdots<i p} \sigma_{i 1} \ldots \sigma_{i p},
\end{gathered}
$$

for the uniform prior $P(\sigma)=1 / 2^{N}$. The overlap is defined by

$$
M(\beta)=\operatorname{Tr}_{\xi} \int d \boldsymbol{J} P(\boldsymbol{J} \mid \boldsymbol{\xi}) P(\boldsymbol{J}) \xi_{i} \operatorname{sgn}\left\langle\sigma_{i}\right\rangle_{\beta}
$$

Image restoration
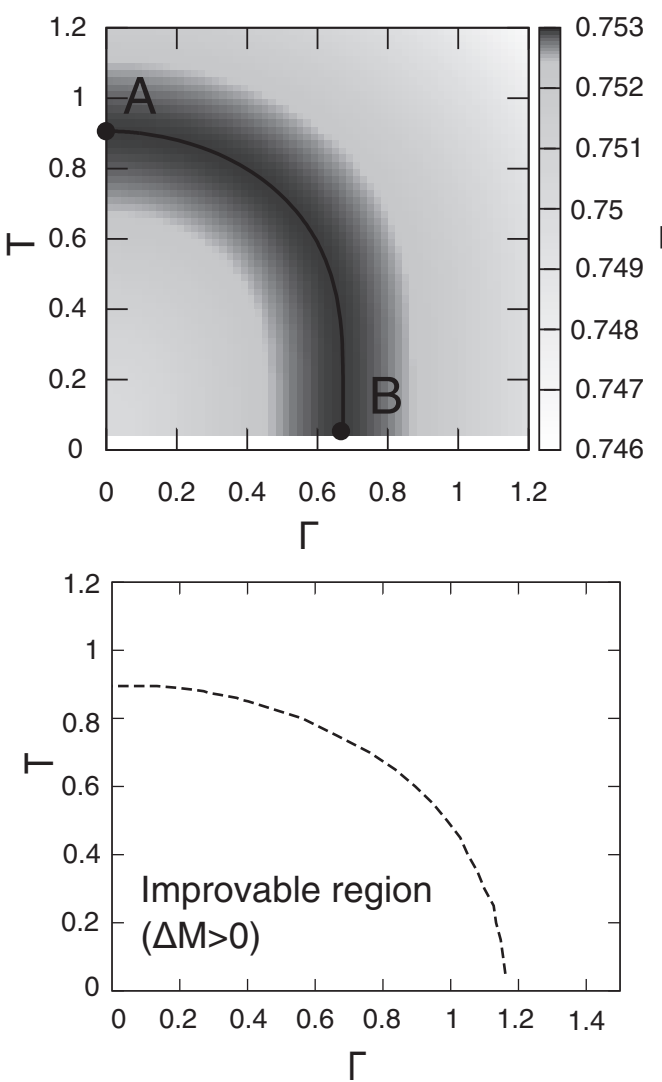

As is the case in image restoration, the overlap is maximized at the Nishimori temperature as

$$
M(\beta) \leqslant M\left(\frac{2 J_{0}}{J^{2}}\right) .
$$

As we saw in Sec. II, we can easily extend the system to the quantum version as

$$
H(\hat{\boldsymbol{\sigma}})=H_{0}\left(\hat{\boldsymbol{\sigma}}^{z}\right)-\Gamma \sum_{i} \hat{\sigma}_{i}^{x} .
$$

The explicit representations of the overlaps in image restoration and Sourlas code have been obtained in previous studies $[7,8]$.

We have presented the value of overlap in the $\Gamma-T$ diagram with gradation in Fig. 8 (upper two panels) and the critical line on which improvable and worsened regions are separated in Fig. 8 (lower two panels). The solid lines in the upper two panels indicate the lines on which overlap is maximized. A peak appears in the overlap and then the location of the peak is roughly the same as that in the results for the classical case. However, detailed investigations revealed that the value of the peak itself for the quantum case was slightly lower than that of the classical decoding for Sourlas code and the CDMA multiuser demodulator. It should be noted that $\Delta M_{A B}\left(=M_{A}-M_{B}\right)$ is 0.0003 for Sourlas code, where $M_{A}$

\section{Sourlas code}
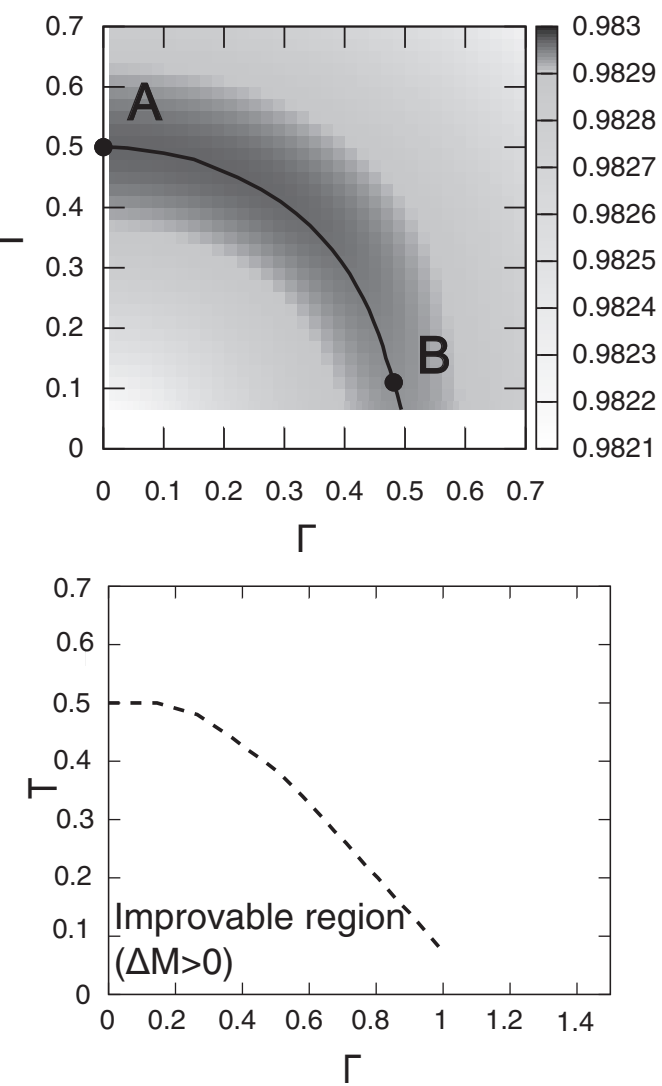

FIG. 8. Upper two panels indicate value of overlap as a function of controlled parameters, temperature, and strength of transverse field for image restoration (left) and Sourlas code (right). Overlap is maximum on solid lines. Lower two panels indicate improvable and worsened regions for image restoration (left) and Sourlas code (right). $\Delta M=0$ holds on dashed lines. We set the parameters to $\tau_{0}=\tau=1.0, h=0.9$, and $\beta_{0}=0.9$ for the image restoration model and $J=J_{0}=1.0$, and $p=3$ for the Sourlas code following previous studies [7,8]. 
and $M_{B}$ represent overlap at points $\mathrm{A}$ and $\mathrm{B}$ in the upper panels of Fig. 8. The peak of the overlap for image restoration, on the other hand, does not change within the accuracy of our numerical calculations, i.e., $\Delta M_{A B}$ is smaller than $10^{-5}$ in our calculations.

The dashed lines in the lower two panels of Fig. 8 indicate the border lines on which $\Delta M=0$ holds. Hence, the $\Delta M$ defined by Eq. (52) is regarded as a "barometer" to check whether decoding with the transverse field is improved in comparison with that in the classical case. We confirmed from these results that the properties of the QMPM estimate follow remarks 1 and 2 that were previously mentioned.

We also note that the maximum average-case performance with the transverse field cannot exceed the classical one because the classical channel and classical bits were only considered. If the channel noise and original bit sequences are chosen as the quantum-mechanical process and qubits respectively, the QMPM estimate would be effective because one can tune the hyperparameters so as to make the posterior identical to the corresponding true distribution.

Although we drew these conclusions from our limited applications of the transverse field, some issues still remain that need to be resolved. We actually used several approximations, replica symmetries (RSs), and static approximations (SAs) in our analysis. As these approximations may be naturally expected to be broken in the low temperature region, we should draw a so-called de Almeida-Thouless (AT) line [22], and we should also discuss the validity of SA. Although the validity of SA has been partially investigated in the quantum random energy model [23], it has not yet been investigated for Ising spin glass in a transverse field. It is generally very difficult to carry out numerical calculations involving very low temperatures with reliable numerical accuracy, and pure quantum demodulation, which is defined as QMPM without any thermal fluctuations, is also very difficult to address. Apart from these perspectives, compressed sensing (CS) is now becoming a hot topic as an effective technique to understand signals from some observable data in various engineering fields, as we stated in Sec. I. Obviously, our formulation using the transverse field could be applied to CS and this should be addressed in future studies.

\section{ACKNOWLEDGMENTS}

This work was partially supported by Grants-in-Aid for Scientific Research (Grants No. 2512009, No. 25330283, No. 2512001313, and No. 2533027803) and that by the Japan Society for the Promotion of Science (JSPS) Fellows (Grant No. 12J06501) from the Ministry of Education, Culture, Sports, Science and Technology of Japan.

\section{APPENDIX: DERIVATION OF FREE ENERGY}

We derive the explicit expression for free energy (38) in this Appendix. We first introduce the following transformation:

$$
u_{0}^{k}=\frac{1}{\sqrt{N}} \sum_{i=1}^{N} \eta_{i}^{k} \xi_{i}, \quad u_{\mu}^{k}(t)=\frac{1}{\sqrt{N}} \sum_{i=1}^{N} \eta_{i}^{k} \sigma_{i}^{\mu}(t)
$$

where $k$ stands for the spreading code index, $\mu$ denotes the replica index, $t$ represents the Trotter index, and $i$ is the spatial index. Then, we write the partition function (34) in terms of the Fourier transform as

$$
\begin{aligned}
{\left[Z^{n}\right]=} & \frac{1}{2^{N}} \frac{1}{2^{N K}} \sum_{\mathbf{H}} \sum_{\left\{\sigma^{k}(s)\right\}} \operatorname{Tr}_{\xi} \operatorname{Tr}_{\left\{\boldsymbol{\sigma}^{\mu}(t)\right\}} \int \prod_{k} \frac{d u_{0}^{k} d \hat{u}_{0}^{k}}{2 \pi} \exp \left[i \hat{u}_{0}^{k}\left(u_{0}^{k}-\frac{1}{\sqrt{N}} \sum_{i} \eta_{i}^{k} \xi_{i}\right)\right] \\
= & \prod_{k} \prod_{\mu, t} \frac{d u_{\mu}^{k}(t) d \hat{u}_{\mu}^{k}(t)}{2 \pi} \exp \left[i \hat{u}_{\mu}^{k}(t)\left(u_{\mu}^{k}(t)-\frac{1}{\sqrt{N}} \sum_{i} \eta_{i}^{k} \sigma_{i}^{\mu}(t)\right)\right] \prod_{k} d y^{k}\left(\frac{\beta_{0}}{2 \pi}\right)^{1 / 2} \exp \left(-\frac{\beta_{0}}{2}\left(y^{k}-u_{0}^{k}\right)^{2}\right) \\
& \times \exp \left[-\frac{\beta}{P} \sum_{\mu, t} \sum_{k}\left(\frac{1}{2}\left[u_{\mu}^{k}(t)\right]^{2}-y^{k} u_{\mu}^{k}(t)\right)\right] \exp \left(B \sum_{\mu, t} \sum_{i} \sigma_{i}^{\mu}(t) \sigma_{i}^{\mu}(t+1)\right) .
\end{aligned}
$$

Here, we carry out the sum of $\mathbf{H}$ in the above expression as

$$
\begin{aligned}
L_{\xi} & \equiv \frac{1}{2^{N K}} \sum_{\mathbf{H}} \prod_{k} \exp \left(-\frac{i \hat{u}_{0}^{k}}{\sqrt{N}} \sum_{i} \eta_{i}^{k} \xi_{i}\right) \prod_{\mu, t} \prod_{k} \exp \left(-\frac{i \hat{u}_{\mu}^{k}(t)}{\sqrt{N}} \sum_{i} \eta_{i}^{k} \sigma_{i}^{\mu}(t)\right) \\
& =\prod_{i, k} \frac{1}{2} \sum_{\eta_{i}^{k}} \exp \left\{-\frac{i \eta_{i}^{k}}{\sqrt{N}}\left(\hat{u}_{0}^{k} \xi_{i}+\sum_{\mu, t} \hat{u}_{\mu}^{k}(t) \sigma_{i}^{\mu}(t)\right)\right\} \\
& =\prod_{i, k} \cos \left\{\frac{1}{\sqrt{N}}\left(\hat{u}_{0}^{k} \xi_{i}+\sum_{\mu, t} \hat{u}_{\mu}^{k}(t) \sigma_{i}^{\mu}(t)\right)\right\}
\end{aligned}
$$




$$
\begin{aligned}
& \simeq \prod_{i, k} \exp \left\{-\frac{1}{2 N}\left(\hat{u}_{0}^{k} \xi_{i}+\sum_{\mu, t} \hat{u}_{\mu}^{k}(t) \sigma_{i}^{\mu}(t)\right)^{2}\right\} \\
& =\prod_{i, k} \exp \left\{-\frac{1}{2 N}\left(\hat{u}_{0}^{k}\right)^{2}-\frac{1}{2 N} \sum_{\mu}\left(\sum_{t} \hat{u}_{\mu}^{k}(t) \sigma_{i}^{\mu}(t)\right)^{2}-\frac{1}{N} \sum_{\mu<\nu} \sum_{t, t^{\prime}} \hat{u}_{\mu}^{k}(t) \hat{u}_{\nu}^{k}\left(t^{\prime}\right) \sigma_{i}^{\mu}(t) \sigma_{i}^{\nu}\left(t^{\prime}\right)-\frac{1}{N} \hat{u}_{0}^{k} \xi_{i} \sum_{\mu, t} \hat{u}_{\mu}^{k}(t) \sigma_{i}^{\mu}(t)\right\} .
\end{aligned}
$$

By introducing the order parameters defined by (35)-(37), we have

$$
\begin{aligned}
L_{\xi}= & \int \prod_{\mu, t} d R_{\mu}(t) \delta\left(R_{\mu}(t)-\frac{1}{N} \sum_{i} \xi_{i} \sigma_{i}^{\mu}(t)\right) \prod_{\mu, t, t^{\prime}} d Q_{\mu \mu}\left(t, t^{\prime}\right) \delta\left(Q_{\mu \mu}\left(t, t^{\prime}\right)-\frac{1}{N} \sum_{i} \sigma_{i}^{\mu}(t) \sigma_{i}^{\mu}\left(t^{\prime}\right)\right) \\
& \times \prod_{\mu<v, t, t^{\prime}} d Q_{\mu v}\left(t, t^{\prime}\right) \delta\left(Q_{\mu \nu}\left(t, t^{\prime}\right)-\frac{1}{N} \sum_{i} \sigma_{i}^{\mu}(t) \sigma_{i}^{v}\left(t^{\prime}\right)\right) \exp \left\{-\frac{1}{2} \sum_{k}\left(\hat{u}_{0}^{k}\right)^{2}\right. \\
& \left.-\frac{1}{2} \sum_{\mu, k} \sum_{t, t^{\prime}} \hat{u}_{\mu}^{k}(t) \hat{u}_{\mu}^{k}\left(t^{\prime}\right) Q_{\mu \mu}\left(t, t^{\prime}\right)-\sum_{\mu<v} \sum_{t, t^{\prime}, k} \hat{u}_{\mu}^{k}(t) \hat{u}_{v}^{k}\left(t^{\prime}\right) Q_{\mu \nu}\left(t, t^{\prime}\right)-\sum_{k} \hat{u}_{0}^{k} \sum_{\mu, t} \hat{u}_{\mu}^{k}(t) R_{\mu}(t)\right\} .
\end{aligned}
$$

From the above calculations, we rewrite the partition function (A2) as

$$
\begin{aligned}
{\left[Z^{n}\right]=} & \int\left(\prod_{\mu, t} \frac{N i d \hat{R}_{\mu}(t) d R_{\mu}(t)}{2 \pi}\right)\left(\prod_{\mu, t, t^{\prime}} \frac{N i d \hat{Q}_{\mu \mu}\left(t, t^{\prime}\right) d Q_{\mu \mu}\left(t, t^{\prime}\right)}{2 \pi}\right)\left(\prod_{\mu<\nu, t, t^{\prime}} \frac{N i d \hat{Q}_{\mu \nu}\left(t, t^{\prime}\right) d Q_{\mu \nu}\left(t, t^{\prime}\right)}{2 \pi}\right) e^{N\left(g_{1}+g_{2}+g_{3}\right)}, \\
e^{N g_{1}}= & \int\left(\prod_{k} \frac{d u_{0}^{k} d \hat{u}_{0}^{k}}{2 \pi}\right)\left(\prod_{k, \mu, t} \frac{d u_{\mu}^{k}(t) d \hat{u}_{\mu}^{k}(t)}{2 \pi}\right)\left[\prod_{k} d y^{k}\left(\frac{\beta_{0}}{2 \pi}\right)^{1 / 2}\right] \exp \left\{-\frac{\beta_{0}}{2} \sum_{k}\left(y^{k}-u_{0}^{k}\right)^{2}\right. \\
& -\sum_{k} \hat{u}_{0}^{k} \sum_{\mu, t} \hat{u}_{\mu}^{k}(t) R_{\mu}(t)-\frac{1}{2} \sum_{\mu, k} \sum_{t, t^{\prime}} \hat{u}_{\mu}^{k}(t) \hat{u}_{\mu}^{k}\left(t^{\prime}\right) Q_{\mu \mu}\left(t, t^{\prime}\right)-\sum_{\mu<v} \sum_{t, t^{\prime}, k} \hat{u}_{\mu}^{k}(t) \hat{u}_{v}^{k}\left(t^{\prime}\right) Q_{\mu \nu}\left(t, t^{\prime}\right) \\
& \left.-\frac{1}{2} \sum_{k}\left(\hat{u}_{0}^{k}\right)^{2}+i \sum_{k} u_{0}^{k} \hat{u}_{0}^{k}+i \sum_{k, \mu, t} u_{\mu}^{k}(t) \hat{u}_{\mu}^{k}(t)-\frac{\beta}{2 P} \sum_{\mu, t, k}\left\{\left[u_{\mu}^{k}(t)\right]^{2}-2 u_{\mu}^{k}(t) y^{k}\right\}\right\}, \\
e^{N g_{2}}= & \operatorname{Tr}_{\xi} P(\xi) \underset{\left\{\sigma^{\mu}(t)\right\}}{\operatorname{Tr}} \exp \left\{\frac{1}{P} \sum_{\mu, t} \hat{R}_{\mu}(t) \sum_{i} \xi_{i} \sigma_{i}^{\mu}(t)+\frac{1}{P^{2}} \sum_{\mu, t, t^{\prime}} \hat{Q}_{\mu \mu}\left(t, t^{\prime}\right) \sum_{i} \sigma_{i}^{\mu}(t) \sigma_{i}^{\mu}\left(t^{\prime}\right)\right. \\
& \left.+\frac{1}{P^{2}} \sum_{\mu<\nu, t, t^{\prime}} \hat{Q}_{\mu \nu}\left(t, t^{\prime}\right) \sum_{i} \sigma_{i}^{\mu}(t) \sigma_{i}^{\nu}\left(t^{\prime}\right)+B \sum_{t, \mu} \sum_{i} \sigma_{i}^{\mu}(t) \sigma_{i}^{\mu}(t+1)\right\} \\
e^{N g_{3}}= & \exp \left\{-\frac{N}{P} \sum_{\mu, t} \hat{R}_{\mu}(t) R_{\mu}(t)-\frac{N}{P^{2}} \sum_{\mu, t, t^{\prime}} \hat{Q}_{\mu \mu}\left(t, t^{\prime}\right) Q_{\mu \mu}\left(t, t^{\prime}\right)-\frac{N}{P^{2}} \sum_{\mu<v, t, t^{\prime}} \hat{Q}_{\mu \nu}\left(t, t^{\prime}\right) Q_{\mu \nu}\left(t, t^{\prime}\right)\right\} .
\end{aligned}
$$

In the following, we calculate $e^{N g_{1}}, e^{N g_{2}}$, and $e^{N g_{3}}$, and then derive the free energy of the CDMA model with the transverse field.

By integrating $u_{0}^{k}$ and $\hat{u}_{0}^{k}$ in Eq. (A10), $e^{N g_{1}}$ can be expressed as

$$
\begin{aligned}
e^{N g_{1}}= & \int\left(\prod_{k, \mu, t} \frac{d u_{\mu}^{k}(t) d \hat{u}_{\mu}^{k}(t)}{2 \pi}\right)\left(\prod_{k} d y^{k} \frac{1}{2 \pi} \sqrt{\frac{2 \beta_{0} \pi}{1+\beta_{0}}}\right) \exp \left\{-\frac{1}{2} \sum_{\mu, k} \sum_{t, t^{\prime}} \hat{u}_{\mu}^{k}(t) \hat{u}_{\mu}^{k}\left(t^{\prime}\right) Q_{\mu \mu}\left(t, t^{\prime}\right)\right. \\
& -\sum_{\mu<v} \sum_{t, t^{\prime}, k} \hat{u}_{\mu}^{k}(t) \hat{u}_{\nu}^{k}\left(t^{\prime}\right) Q_{\mu \nu}\left(t, t^{\prime}\right)+i \sum_{k, \mu, t} u_{\mu}^{k}(t) \hat{u}_{\mu}^{k}(t)-\frac{\beta}{2 P} \sum_{\mu, t, k}\left\{\left[u_{\mu}^{k}(t)\right]^{2}-2 u_{\mu}^{k}(t) y^{k}\right\} \\
& \left.-\frac{\beta_{0}}{2\left(1+\beta_{0}\right)} \sum_{k}\left(y^{k}\right)^{2}-\frac{i \beta_{0}}{1+\beta_{0}} \sum_{k} y^{k} \sum_{\mu, t} \hat{u}_{\mu}^{k}(t) R_{\mu}(t)+\frac{\beta_{0}}{2\left(1+\beta_{0}\right)} \sum_{k}\left(\sum_{\mu, t} \hat{u}_{\mu}^{k}(t) R_{\mu}(t)\right)^{2}\right\} .
\end{aligned}
$$


The integration over $y^{k}$ in the above equation can be carried out as

$$
\begin{aligned}
& \int\left(\prod_{k} d y^{k}\right) \exp \left(\frac{\beta}{P} \sum_{\mu, k, t} u_{\mu}^{k}(t) y^{k}-\frac{\beta_{0}}{2\left(1+\beta_{0}\right)} \sum_{k}\left(y^{k}\right)^{2}-\frac{i \beta_{0}}{1+\beta_{0}} \sum_{k} y^{k} \sum_{\mu, t} \hat{u}_{\mu}^{k}(t) R_{\mu}(t)\right) \\
& =\int \prod_{k} d y^{k} \exp \left[-\frac{\beta_{0}}{2\left(1+\beta_{0}\right)}\left(y^{k}\right)^{2}+\left(\frac{\beta}{P} \sum_{\mu, t} u_{\mu}^{k}(t)-\frac{i \beta_{0}}{1+\beta_{0}} \sum_{\mu, t} \hat{u}_{\mu}^{k}(t) R_{\mu}(t)\right) y^{k}\right] \\
& =\prod_{k} \sqrt{\frac{2\left(1+\beta_{0}\right) \pi}{\beta_{0}}} \exp \left[\frac{1+\beta_{0}}{2 \beta_{0}}\left(\frac{\beta}{P} \sum_{\mu, t} u_{\mu}^{k}(t)-\frac{i \beta_{0}}{1+\beta_{0}} \sum_{\mu, t} \hat{u}_{\mu}^{k}(t) R_{\mu}(t)\right)^{2}\right] \\
& =\prod_{k} \sqrt{\frac{2\left(1+\beta_{0}\right) \pi}{\beta_{0}}} \exp \left\{\frac{\beta^{2}\left(1+\beta_{0}\right)}{2 \beta_{0} P^{2}}\left(\sum_{\mu, t} u_{\mu}^{k}(t)\right)^{2}-\frac{i \beta}{P}\left(\sum_{\mu, t} u_{\mu}^{k}(t)\right)\left(\sum_{\mu, t} \hat{u}_{\mu}^{k}(t) R_{\mu}(t)\right)-\frac{\beta_{0}}{2\left(1+\beta_{0}\right)}\left(\sum_{\mu, t} \hat{u}_{\mu}^{k}(t) R_{\mu}(t)\right)^{2}\right\} .
\end{aligned}
$$

The integration over $u_{\mu}^{k}(t)$ can be carried out independently for each $k$ as

$$
\begin{aligned}
\prod_{k} \int & \left(\prod_{\mu, t} d u_{\mu}^{k}(t)\right) \exp \left\{i \sum_{\mu, t} u_{\mu}^{k}(t) \hat{u}_{\mu}^{k}(t)-\frac{\beta}{2 P} \sum_{\mu, t}\left[u_{\mu}^{k}(t)\right]^{2}+\frac{\beta^{2}\left(1+\beta_{0}\right)}{2 \beta_{0} P^{2}}\left(\sum_{\mu, t} u_{\mu}^{k}(t)\right)^{2}\right. \\
& \left.-\frac{i \beta}{P}\left(\sum_{\mu, t} u_{\mu}^{k}(t)\right)\left(\sum_{\mu, t} \hat{u}_{\mu}^{k}(t) R_{\mu}(t)\right)\right\} \\
= & \prod_{k} \int D a\left(\prod_{\mu, t} d u_{\mu}^{k}(t)\right) \exp \left\{\frac{a \beta}{P} \sqrt{\frac{1+\beta_{0}}{\beta_{0}}} \sum_{\mu, t} u_{\mu}^{k}(t)+i \sum_{\mu, t} u_{\mu}^{k}(t) \hat{u}_{\mu}^{k}(t)-\frac{\beta}{2 P} \sum_{\mu, t}\left[u_{\mu}^{k}(t)\right]^{2}\right. \\
& \left.-\frac{i \beta}{P}\left(\sum_{\mu, t} u_{\mu}^{k}(t)\right)\left(\sum_{\mu, t} \hat{u}_{\mu}^{k}(t) R_{\mu}(t)\right)\right\} \\
= & \prod_{k} \int D a \prod_{\mu, t} \sqrt{\frac{2 P \pi}{\beta}} \exp \left\{\frac{P}{2 \beta}\left(\frac{a \beta}{P} \sqrt{\frac{1+\beta_{0}}{\beta_{0}}}+i \hat{u}_{\mu}^{k}(t)-\frac{i \beta}{P} \sum_{\nu, t^{\prime}} \hat{u}_{\nu}^{k}\left(t^{\prime}\right) R_{v}\left(t^{\prime}\right)\right)^{2}\right\} \\
= & \prod_{k} \int D a \prod_{\mu, t} \sqrt{\frac{2 P \pi}{\beta}} \exp \left\{\frac{a^{2} \beta\left(1+\beta_{0}\right)}{2 P \beta_{0}}-\frac{P}{2 \beta}\left[\hat{u}_{\mu}^{k}(t)\right]^{2}-\frac{\beta}{2 P}\left(\sum_{v, t^{\prime}} \hat{u}_{v}^{k}\left(t^{\prime}\right) R_{\nu}\left(t^{\prime}\right)\right)^{2}\right. \\
& \left.+i a \sqrt{\frac{1+\beta_{0}}{\beta_{0}}} \hat{u}_{\mu}^{k}(t)+\left(\sum_{\nu, t^{\prime}} \hat{u}_{v}^{k}\left(t^{\prime}\right) R_{\nu}\left(t^{\prime}\right)\right) \hat{u}_{\mu}^{k}(t)-\frac{i a \beta}{P} \sqrt{\frac{1+\beta_{0}}{\beta_{0}}}\left(\sum_{v, t^{\prime}} \hat{u}_{v}^{k}\left(t^{\prime}\right) R_{v}\left(t^{\prime}\right)\right)\right\},
\end{aligned}
$$

when we apply Hubbard-Stratonovich transformation,

$$
\exp \left(\frac{x^{2}}{2}\right)=\int D a e^{a x}, \quad\left(D a=\frac{d a e^{-a^{2} / 2}}{\sqrt{2 \pi}}\right),
$$

to the term $\left[u_{\mu}^{k}(t)\right]^{2}$ in Eq. (A17).

Here, we assume replica symmetry (RS) and static approximation (SA) [24]:

$$
\begin{array}{lll}
R_{\mu}(t)=R, & Q_{\mu \nu}\left(t, t^{\prime}\right)=q, & Q_{\mu \mu}\left(t, t^{\prime}\right)=\chi\left(t \neq t^{\prime}\right), \\
\hat{R}_{\mu}(t)=\hat{R}, & \hat{Q}_{\mu \nu}\left(t, t^{\prime}\right)=\hat{q}, & \hat{Q}_{\mu \mu}\left(t, t^{\prime}\right)=\hat{\chi} .
\end{array}
$$

Note that the following relation exits for $Q_{\mu \mu}(t, t)=1$ under RS and SA:

$$
\sum_{k, \mu} \sum_{t, t^{\prime}} \hat{u}_{\mu}^{k}(t) \hat{u}_{\mu}^{k}\left(t^{\prime}\right) Q_{\mu \mu}\left(t, t^{\prime}\right) \rightarrow \chi \sum_{k, \mu} \sum_{t, t^{\prime}} \hat{u}_{\mu}^{k}(t) \hat{u}_{\mu}^{k}\left(t^{\prime}\right)-(\chi-1) \sum_{k, \mu, t}\left[\hat{u}_{\mu}^{k}(t)\right]^{2} .
$$


By using the Hubbard-Stratonovich transformation on $\left(\sum_{\mu} \sum_{t}\right)^{2}$ and $\sum_{\mu}\left(\sum_{t}\right)^{2}$ and bearing in mind the order of the product of indices, $k, \mu$, and $t$, we have

$$
\begin{aligned}
e^{N g_{1}}= & \prod_{k} \int D a \int D b \prod_{\mu} \int D c \prod_{t} \frac{d \hat{u}_{\mu}^{k}(t)}{2 \pi} \sqrt{\frac{2 P \pi}{\beta}} \exp \left[\frac{a^{2} \beta\left(1+\beta_{0}\right)}{2 P \beta_{0}}-\frac{P-\beta(\chi-1)}{2 \beta}\left[\hat{u}_{\mu}^{k}(t)\right]^{2}\right. \\
& \left.+\left\{a i \sqrt{\frac{1+\beta_{0}}{\beta_{0}}}(1-n \beta R)+b \sqrt{2 R-q-n \beta R^{2}}+c \sqrt{q-\chi}\right\} \hat{u}_{\mu}^{k}(t)\right] \\
= & \prod_{k} \int D a \int D b \exp \left(\frac{n a^{2} \beta\left(1+\beta_{0}\right)}{2 \beta_{0}}\right) \prod_{\mu} \int D c \prod_{t} \frac{1}{2 \pi} \sqrt{\frac{2 P \pi}{\beta}} \sqrt{\frac{2 \beta \pi}{P-\beta(\chi-1)}} \\
& \times \exp \left\{\frac{\beta}{2 P}\left(a i \sqrt{\frac{1+\beta_{0}}{\beta_{0}}}(1-n \beta R)+b \sqrt{2 R-q-n \beta R^{2}}+c \sqrt{q-\chi}\right)^{2}\right\} .
\end{aligned}
$$

Here, we introduce the following replacements to simplify the above equation:

$$
\begin{aligned}
A & \equiv i \sqrt{\frac{1+\beta_{0}}{\beta_{0}}}(1-n \beta R), \\
B & \equiv \sqrt{2 R-q-n \beta R^{2}}, \\
C & \equiv \sqrt{q-\chi}, \\
X_{a b} & \equiv A a+B b .
\end{aligned}
$$

The coefficient under the condition that $P \rightarrow \infty$ can be transformed as

$$
\begin{aligned}
\prod_{t} \sqrt{\frac{P}{P-\beta(\chi-1)}} & \simeq \prod_{t}\left\{1+\frac{\beta}{2 P}(\chi-1)\right\} \\
& \simeq \prod_{t} \exp \ln \left\{1+\frac{\beta}{2 P}(\chi-1)\right\} \\
& \simeq \exp \left(\frac{\beta}{2}(\chi-1)\right) .
\end{aligned}
$$

Then, we carry out integrations over $a, b$, and $c$ as

$$
\begin{aligned}
e^{N g_{1}} & =\prod_{k} \int D a \int D b \exp \left(\frac{n a^{2} \beta\left(1+\beta_{0}\right)}{2 \beta_{0}}+\frac{n \beta}{2}(\chi-1)\right) \prod_{\mu} \int D c \exp \left(\frac{\beta}{2}\left(X_{a b}+C c\right)^{2}\right) \\
& =\prod_{k} \exp \left(\frac{n \beta}{2}(\chi-1)\right)\left(\frac{1}{1-\beta C^{2}}\right)^{n / 2} \int D a \int D b \exp \left\{\frac{n \beta}{2}\left(\frac{1+\beta_{0}}{\beta_{0}} a^{2}+\frac{X_{a b}^{2}}{1-\beta C^{2}}\right)\right\} \\
& \simeq \prod_{k} \exp \left(\frac{n \beta}{2}(\chi-1)\right)\left(\frac{1}{1-\beta C^{2}}\right)^{n / 2} \int D a \int D b\left\{1+\frac{n \beta}{2}\left(\frac{1+\beta_{0}}{\beta_{0}} a^{2}+\frac{X_{a b}^{2}}{1-\beta C^{2}}\right)\right\} \\
& =\prod_{k} \exp \left(\frac{n \beta}{2}(\chi-1)\right)\left(\frac{1}{1-\beta C^{2}}\right)^{n / 2}\left\{1+\frac{\beta n}{2}\left(\frac{1+\beta_{0}}{\beta_{0}}+\frac{A^{2}+B^{2}}{1-\beta C^{2}}\right)\right\} \\
& =\prod_{k} \exp \left(\frac{n \beta}{2}(\chi-1)\right)\left(\frac{1}{1-\beta C^{2}}\right)^{n / 2}\left\{1+\frac{\beta n}{2}\left(\frac{1+\beta_{0}}{\beta_{0}}+\frac{2 R-q-\left(1+\beta_{0}^{-1}\right)}{1-\beta(q-\chi)}\right)+O\left(n^{2}\right)\right\} \\
& \simeq \prod_{k} \exp \left[\frac{n}{2}\left\{-\ln [1-\beta(q-\chi)]+\frac{\beta\left(1+\beta_{0}\right)}{\beta_{0}}+\frac{\beta\left[2 R-q-\left(1+\beta_{0}^{-1}\right)\right]}{1-\beta(q-\chi)}+\beta(\chi-1)\right\}\right] .
\end{aligned}
$$

For $\alpha \equiv K / N$, we derive the final expression of $g_{1}$ as

$$
\frac{g_{1}}{n} \simeq \frac{\alpha}{2}\left\{-\ln [1-\beta(q-\chi)]+\frac{\beta\left(1+\beta_{0}\right)}{\beta_{0}}+\frac{\beta\left[2 R-q-\left(1+\beta_{0}^{-1}\right)\right]}{1-\beta(q-\chi)}+\beta(\chi-1)\right\} .
$$


We next calculate $e^{\mathrm{Ng}_{2}}$ under RS and SA conditions as

$$
\begin{aligned}
e^{N g_{2}=} & \operatorname{Tr}_{\xi} P(\xi) \operatorname{Tr}_{\left\{\sigma^{\mu}(t)\right\}} \exp \left\{\frac{\hat{R}}{P} \sum_{\mu, t} \sum_{i} \xi_{i} \sigma_{i}^{\mu}(t)+\frac{\hat{\chi}}{P^{2}} \sum_{\mu, t, t^{\prime}} \sum_{i} \sigma_{i}^{\mu}(t) \sigma_{i}^{\mu}\left(t^{\prime}\right)\right. \\
& \left.+\frac{\hat{q}}{2 P^{2}} \sum_{i}\left(\sum_{\mu, t} \sigma_{i}^{\mu}(t)\right)^{2}-\frac{\hat{q}}{2 P^{2}} \sum_{i} \sum_{\mu}\left(\sum_{t} \sigma_{i}^{\mu}(t)\right)^{2}+B \sum_{t, \mu} \sum_{i} \sigma_{i}^{\mu}(t) \sigma_{i}^{\mu}(t+1)\right\} \\
= & \frac{1}{2^{N}} \operatorname{Tr}_{\xi} \operatorname{Tr}_{\left\{\sigma^{\mu}(t)\right\}} \prod_{i} \int D w \exp \left(\frac{w \sqrt{\hat{q}}}{P} \sum_{\mu, t} \sigma_{i}^{\mu}(t)\right) \prod \prod_{\mu} \int D z \exp \left(\frac{z \sqrt{2 \hat{\chi}-\hat{q}}}{P} \sum_{t} \sigma_{i}^{\mu}(t)\right) \\
& \times \exp \left\{\frac{\hat{R}}{P} \sum_{t} \sum_{i} \xi_{i} \sigma_{i}^{\mu}(t)+B \sum_{t} \sigma_{i}^{\mu}(t) \sigma_{i}^{\mu}(t+1)\right\} \\
= & \prod_{i} \frac{1}{2} \operatorname{Tr}_{\xi_{i}} \int D w \exp \left(\frac{w \sqrt{\hat{q}}}{P} \sum_{\mu, t} \sigma_{i}^{\mu}(t)\right) \prod_{\mu} \int D z \exp \left(\frac{z \sqrt{2 \hat{\chi}-\hat{q}}}{P} \sum_{t} \sigma_{i}^{\mu}(t)\right) \\
& \times \operatorname{Tr}_{\sigma_{i}^{\mu}(t)} \exp \left\{\frac{\hat{R}}{P} \sum_{t} \sum_{i} \xi_{i} \sigma_{i}^{\mu}(t)+B \sum_{t} \sigma_{i}^{\mu}(t) \sigma_{i}^{\mu}(t+1)\right\} \\
= & \prod_{i} \frac{1}{2} \operatorname{Tr}_{\xi_{i}} \int D w \prod_{\mu} \int D z \operatorname{Tr}_{\sigma_{i}^{\mu}(t)} \exp \left\{\frac{z \sqrt{2 \hat{\chi}-\hat{q}}+w \sqrt{\hat{q}}+\hat{R} \xi_{i}}{P} \sum_{t} \sigma_{i}^{\mu}(t)+B \sum_{t} \sigma_{i}^{\mu}(t) \sigma_{i}^{\mu}(t+1)\right\} .
\end{aligned}
$$

By applying ST decomposition to the above equation, we can take a spin trace as

$$
\begin{aligned}
e^{N g_{2}} & \rightarrow \prod_{i} \frac{1}{2} \operatorname{Tr}_{\xi_{i}} \int D w \prod_{\mu} \int D z \operatorname{Tr} \exp \left\{\left(z \sqrt{2 \hat{\chi}-\hat{q}}+w \sqrt{\hat{q}}+\hat{R} \xi_{i}\right) \hat{\sigma}_{i}^{z}+\beta \Gamma \hat{\sigma}_{i}^{x}\right\} \\
& =\prod_{i} \frac{1}{2} \operatorname{Tr}_{\xi_{i}} \int D w \prod_{\mu} \int D z 2 \cosh \sqrt{\Phi\left(\xi_{i}\right)^{2}+\beta^{2} \Gamma^{2}} \\
& =\prod_{i} \frac{1}{2} \operatorname{Tr}_{\xi_{i}} \int D w\left(\int D z 2 \cosh \sqrt{\Phi\left(\xi_{i}\right)^{2}+\beta^{2} \Gamma^{2}}\right)^{n} \\
& \simeq \prod_{i} \int D w\left(\int D z 2 \cosh \sqrt{\Phi^{2}+\beta^{2} \Gamma^{2}}\right)^{n} \\
& \simeq \exp N \ln \left\{1+n \int D z \ln \left(\int D w 2 \cosh \sqrt{\Phi^{2}+\beta^{2} \Gamma^{2}}\right)\right\} \\
& =\exp N n \int D z \ln \left(\int D w 2 \cosh \sqrt{\Phi^{2}+\beta^{2} \Gamma^{2}}\right)
\end{aligned}
$$

where

$$
\Phi\left(\xi_{i}\right)=z \sqrt{2 \hat{\chi}-\hat{q}}+w \sqrt{\hat{q}}+\hat{R} \xi_{i}
$$

Therefore, we obtain

$$
\begin{aligned}
\frac{g_{2}}{n} & \simeq \int D z \ln \left(\int D w 2 \cosh \sqrt{\Phi^{2}+\beta^{2} \Gamma^{2}}\right), \\
\Phi & =w \sqrt{2 \hat{\chi}-\hat{q}}+z \sqrt{\hat{q}}+\hat{R} .
\end{aligned}
$$

We finally calculate $e^{N g_{3}}$ under RS and SA conditions as

$$
e^{N g_{3}} \simeq \exp \left\{n N\left(-\hat{R} R-\hat{\chi} \chi+\frac{\hat{q} q}{2}\right)\right\} .
$$

Thus, we obtain the following form:

$$
\frac{g_{3}}{n} \simeq-\hat{R} R-\hat{\chi} \chi+\frac{\hat{q} q}{2} .
$$


From Eqs. (A34), (A46), and (A48), we obtain the free energy density as

$$
\begin{aligned}
-\beta f= & \frac{g_{1}}{n}+\frac{g_{2}}{n}+\frac{g_{3}}{n} \\
= & \frac{\alpha}{2}\left\{-\ln [1-\beta(q-\chi)]+\frac{\beta\left(1+\beta_{0}\right)}{\beta_{0}}+\frac{\beta\left[2 R-q-\left(1+\beta_{0}^{-1}\right)\right]}{1-\beta(q-\chi)}+\beta(\chi-1)\right\}-\hat{R} R \\
& -\hat{\chi} \chi+\frac{\hat{q} q}{2}+\int D z \ln \left(\int D w 2 \cosh \sqrt{\Phi^{2}+\beta^{2} \Gamma^{2}}\right), \\
\Phi= & \frac{\phi}{\beta}=w \sqrt{2 \hat{\chi}-\hat{q}}+z \sqrt{\hat{q}}+\hat{R} .
\end{aligned}
$$

Considering the classical limits $\Gamma=0$ and $\chi=1$, the classical free energy is recovered.

[1] T. Kadowaki and H. Nishimori, Phys. Rev. E 58, 5355 (1998).

[2] E. Farhi, J. Goldstone, S. Gutmann, J. Lapan, A. Lundgren, and D. Preda, Science 292, 472 (2001).

[3] G. E. Santoro, R. Martonak, E. Tosatti, and R. Car, Science 295, 5564 (2002).

[4] R. Harris, A. J. Berkley, M. W. Johnson, P. Bunyk, S. Govorkov, M. C. Thom, S. Uchaikin, A. B. Wilson, J. Chung, E. Holtham, J. D. Biamonte, A. Yu. Smirnov, M. H. S. Amin, and A. Maassen van den Brink, Phys. Rev. Lett. 98, 177001 (2007).

[5] M. W. Johnson, M. H. S. Amin, S. Gildert, T. Lanting, F. Hamze, N. Dickson, R. Harris, A. J. Berkley, J. Johansson, P. Bunyk, E. M. Chapple, C. Enderud, J. P. Hilton, K. Karimi, E. Ladizinsky, N. Ladizinsky, T. Oh, I. Perminov, C. Rich, M. C. Thom, E. Tolkacheva, C. J. S. Truncik, S. Uchaikin, J. Wang, B. Wilson, and G. Rose, Nature (London) 473, 194 (2011).

[6] S. Boixo, T. Albash, F. M. Spedalieri, N. Chancellor, and D. A. Lidar, Nat. Commun. 4, 3067 (2013).

[7] J. I. Inoue, Phys. Rev. E 63, 046114 (2001).

[8] Y. Otsubo, J. I. Inoue, K. Nagata, and M. Okada, Phys. Rev. E 86, 051138 (2012).

[9] D. Sherrington and S. Kirkpatrick, Phys. Rev. Lett. 35, 1792 (1975).

[10] N. Sourlas, Nature (London) 339, 693 (1989).
[11] J. J. Hopfield, Proc. Natl. Acad. Sci. USA 79, 2554 (1982).

[12] H. Nishimori, Statistical Physics of Spin Glasses and Information Processing (Oxford Science, Oxford, 2001).

[13] T. Tanaka, Europhys. Lett. 54, 540 (2001).

[14] Y. Kabashima, in Proceedings of the IEEE International Symposium on Information Theory, 2003 (IEEE, Piscataway, NJ, 2003), p. 329.

[15] S. Rangan, A. K. Fletcher, and V. K. Goyal, IEEE Trans. Inf. Theory 58, 1902 (2012).

[16] E. J. Candes, J. Romberg, and T. Tao, IEEE Trans. Inf. Theory 52, 489 (2006).

[17] Y. Kabashima, T. Wadayama, and T. Tanaka, J. Stat. Mech. (2009) L09003.

[18] M. Suzuki, Prog. Theor. Phys. 56, 1454 (1976).

[19] D. Thirumalai, Q. Li, and T. R. Kirkpatrick, J. Phys. A 22, 3339 (1989).

[20] M. Yoshida, T. Uezu, T. Tanaka, and M. Okada, J. Phys. Soc. Jpn. 76, 054003 (2007).

[21] H. Nishimori and K. Y. M. Wong, Phys. Rev. E 60, 132 (1999).

[22] J. R. L. de Almeida and D. J. Thouless, J. Phys. A 11, 983 (1978).

[23] T. Obuchi, H. Nishimori, and D. Sherrington, J. Phys. Soc. Jpn. 76, 054002 (2007).

[24] A. J. Bray and M. A. Moore, J. Phys. C 13, L665 (1980). 